\title{
What Time Use Surveys Can (And Cannot) Tell Us about Labor Supply
}

\author{
Cheng Chou* $\quad$ Ruoyao Shi ${ }^{\dagger \ddagger}$ \\ University of Leicester UC Riverside
}

This version: January, 2019

\begin{abstract}
It has been widely acknowledged that the measurement of labor supply in the Current Population Survey (CPS) and other conventional microeconomic surveys has nonclassical measurement error, which will bias the estimates of crucial parameters in labor economics, such as labor supply elasticity. Time diary studies, such as the American Time Use Survey (ATUS), only have accurate measurement of hours worked on a single day, hence the weekly hours worked are unobserved. Despite the missing data problem, we provide several consistent estimators of the parameters in weekly labor supply equation using the information in the time use surveys. The consistency of our estimators does not require more conditions beyond those for a usual two stage least square (2SLS) estimator when the true weekly hours worked are observed. We also show that it is impossible to recover the weekly number of hours worked or its distribution function from time use surveys like the ATUS. In our empirical application we find considerable evidence of nonclassical measurement error in the hours worked in the CPS, and illustrate the consequences of using mismeasured weekly hours worked in empirical studies.
\end{abstract}

Keywords: measurement error, missing data, instrumental variable, asymptotic efficiency, labor supply

JEL codes: C13, C21, C26, C81, J22

\footnotetext{
${ }^{*}$ School of Business, University of Leicester. Address: 1.01 Mallard House, Leicester, LE1 7RH, UK. Email: cchou@le.ac.uk.

${ }^{\dagger}$ Department of Economics, UC Riverside. Address: 3136 Sproul Hall, Riverside, CA, 92521, USA. Email: ruoyao.shi@ucr.edu.

${ }^{\ddagger}$ We thank Scott Carrell, Robert W. Fairlie, Daniel Gutknecht, Jinyong Hahn, Zhipeng Liao, Jesse Matheson, Geert Ridder, Arthur Stone and the participants at the UCR brown bag seminar for helpful comments.
} 


\section{Introduction}

Empirical work in labor supply depends greatly upon data on how much time people spend working. Unfortunately, there is abundant evidence showing that weekly hours worked are poorly measured in frequently used survey data sets such as the Current Population Survey (CPS) and the Panel Study of Income Dynamics (PSID), and that the measurement error is nonclassical (for example, Bound, Brown, Duncan, and Rodgers, 1989). In particular, survey data appear to consistently overestimate hours worked, and the overestimation increases over time (see Bound, Brown, and Mathiowetz, 2001, Section 6.4 for a summary of several studies). This nonclassical measurement error in weekly hours worked may significantly bias the estimation of labor supply parameters.

Many countries have historical or ongoing time use surveys, 1 including the American Time Use Survey (ATUS) and the Dutch Time Use Survey (DTUS).2 Time use surveys typically ask the respondents to write a detailed time diary of all their activities for a prescribed period, which is one day for the ATUS, hence provide arguably more accurate measures of hours worked for that period. For the same respondents in the ATUS or DTUS, we can observe both the mismeasured hours worked from CPS-like questions on hours worked last week or usually worked and the more accurate hours worked from time-diary records. Throughout the paper, we assume that the hours worked in time use surveys are the true hours worked for the prescribed period. This is merely for the simplicity of exposition, because the main results remain to hold if the measurement error of hours worked in time use surveys is classical. 3

The major limitation of time use surveys is that they often only provide information about labor supply for a few days (DTUS is an exception, in which respondents record their activities in seven consecutive days). If we are interested in estimating weekly labor supply equation or assessing and correcting the measurement error in the weekly hours worked from CPS-like surveys, we naturally want to observe the true weekly hours worked, which is missing in the ATUS that provides hours worked only for one day. This creates a missing data problem, and the time specificity of the ATUS and other time use surveys is the crux of this missing data problem.

The main point of this paper is that despite the missing data problem, we can use information from time use surveys to consistently estimate weekly labor supply equation

\footnotetext{
${ }^{1}$ Including Australia, Canada, China, Japan, New Zealand, Pakistan, Russia, the USA and most European countries.

${ }^{2}$ In Dutch, it is called Het Tijdsbestedingsonderzoek (TBO). In this paper we call it DTUS for the consistency with ATUS.

${ }^{3}$ Estimators will have larger standard errors, but classical measurement errors in dependent variables are known to be much less problematic.
} 
under the same condition as if we had observed true weekly hours worked - that is, the presence of valid and relevant instrumental variables (IV). We provide several consistent estimators, and recommend a particular estimator based on their asymptotic efficiency and finite sample stability. However, due to the missing data problem, it is impossible to recover the weekly hours worked or their distribution function. Hence, accurate hours worked for a few days from time use surveys do not directly quantify or correct the measurement error in the weekly hours in common surveys like CPS. This impossibility result highlights the limitation of time use surveys.

To understand this missing data problem, it helps to employ the potential outcome framework often used for program evaluation. The hours worked on each day of a week can be thought of as a potential outcome. Weekly hours worked are the sum of the seven potential outcomes, but time use surveys often provide information for only a few days, but not all (DTUS is an exception). Just like individual treatment effects, that require knowing individual's potential outcomes in both treatment status and control status, cannot be recovered, even from purely random experiment data, individual weekly hours worked cannot be retrieved from data only on a few days without further assumption. By the same argument, the distribution of weekly hours worked is not identified unless the hours worked on each day are independent, which is unlikely.

Labor supply parameters, on the other hand, are often estimated using certain regression function, which is in essence a conditional mean function. Again, just like average treatment effects can be identified using conditional mean functions, many important labor supply parameters can be recovered using time use survey data. We propose a number of estimators, all of which seem practically appealing. But after investigating their performance, both theoretically and empirically, we recommend the one which we name imputed estimator. It gets the name because it involves imputing weekly hours worked from time use survey. The imputed estimator merely adds one simple step to the usual two-stage least squares (2SLS) estimator.

This paper makes a contribution to the literature on nonclassical measurement error. We show how to consistently estimate parameters of interest when the dependent variable has nonclassical measurement error by combining the usage of two data sources with different time frames, e.g. daily ATUS and weekly CPS. Nonclassical measurement error in dependent variables have drawn far less attention than in independent variables. Abrevaya and Hausman (1999) consider a general class of linear index model with error-ridden dependent variable, while the majority of the literature on nonclassical measurement error considers error-ridden independent variables (for example, Hu and Schennach, 2008; Chen, Hong, and Tamer, 2005; $\mathrm{Hu}$ and Sasaki, 2015). It is unclear, however, whether and how the estimator Abrevaya and Hausman (1999) propose would accommodate endogenous independent vari- 
ables. Moreover, the time specificity is a unique feature when one works with the ATUS and the CPS at the same time. In contrast, the above-mentioned papers on error-ridden independent variables all combine the usage of two data sources with the same time frame. Barrett and Hamermesh (2017) acknowledge the potential significance of such time specificity, but they did not delve into its consequences and possible solutions. Examining how to efficiently utilize such data sets as the ATUS is, on its own, an interesting and relevant inquiry in labor economics.

This paper makes a contribution to the empirical labor economics by investigating the impact of using mismeasured hours worked on the estimation of labor supply equations. A good number of empirical labor economics studies look into error-ridden dependent variables in earning equations, probably as a result of the availability of administrative record data. For example, Duncan and Hill (1985) find that nonclassical measurement error in the dependent variable has a sizable impact on parameter estimates by comparing estimates using earnings data from a survey and from an administrative source. Hours worked, on the other hand, receives much less attention as the dependent variable, and it is unclear what their impacts on labor supply parameter estimates are. Without solving the time specificity problem, Barrett and Hamermesh (2017) estimate weekly labor supply elasticities using both the ATUS daily hours and the recalled weekly hours in the CPS and find that the latter yield positively biased elasticities. Utilizing the DTUS, we compare the usual 2SLS estimator using CPS type recalled weekly hours and that using the time diary weekly hours, and show that the CPS type recalled weekly hours result in large bias. The estimators we consider, on the other hand, contain negligible finite sample biases. In addition, the imputed estimator has the smallest finite sample variances and is stable even in fairly small samples.

This paper also contributes to the labor economics literature by making a number of interesting empirical findings on labor supply elasticities. We apply the imputed estimator to a sample of hourly paid workers who were interviewed in both the ATUS and the CPS, and compare the estimated labor supply elasticities given by the imputed estimator using the ATUS data with those using the error-ridden CPS weekly hours. First, we find that the ATUS suggests that married women have substantially higher elasticity at the intensive margin than unmarried women and men, but CPS suggests that the elasticities at the intensive margin of married women, unmarried women and unmarried men are similar. Our results based on the ATUS is interesting because it complements the widely recognized pattern that married women have higher elasticity at the extensive margin than men and unmarried women (Heckman, 1993; Blau and Kahn, 2007; Blundell et al., 1998). Our results further suggest that the apparent similarity between labor supply elasticities of married women and the other groups at the intensive margin, indicated by analysis based on CPStype survey data (Heckman, 1993), might be due to nonclassical measurement error in hours 
worked. Second, the ATUS suggests that wives' earnings have significant negative effects on husband's hours worked, but the CPS shows that this effect is negligible. Third, the ATUS shows that married women' labor supply is not responsive to the number of older children (aged between 5 to 8) but the CPS indicates a strong negative effect. This contrasts to married women's labor supply elasticity with respect to the number of younger children (aged below 5), in which case both the CPS and the ATUS yield similar large negative effects.

To fix the idea, our analytical results will concentrate on the ATUS and DTUS. Because the DTUS records the respondents' activities for the entire week, it serves as a benchmark against which our analytical results can be checked. Since all respondents of the ATUS have completed questionnaires of the CPS, we also have the mismeasured weekly hours worked and all the other variables from the CPS for the same individuals. By comparing the estimation of labor supply equations using the CPS hours worked and using the ATUS hours with our imputed estimator, we can see the consequence of using mismeasured hours worked, and how our method can mitigate the problem.

Although for concreteness we focus on the intra-temporal labor supply elasticities, we believe that the idea is pervasive. For example, Ahmed, Brzozowski, and Crossley (2006) find that substantial measurement errors in recall food consumption data, and they are nonclassical. The estimation of price elasticity will be biased if one used the recalled food consumption as the dependent variable (Sousa, 2014). Surveys like the Expenditure and Food Survey (EFS) in the UK⿴囗⿱一一⿻儿口 4 use diary system to record volumes purchased and expenditure on a range of goods over a short period. These diary-based data are arguably more accurate than recalled food consumption, but there is also similar time specificity issue.

A large part of this paper will be on how to use the daily hours worked in the ATUS to estimate a weekly labor supply equation. But what is the significance of weekly labor supply? Why not, say, monthly, quarterly, or yearly labor supply? The most important reason is that, once we bridge the gap between daily hours and weekly hours, then going from weekly hours to longer time frame only demands the same tools. Therefore, we believe that using the daily hours data in the ATUS to estimate weekly labor supply parameters is a reasonable starting point. Another reason is that the CPS records weekly hours, , and since the ATUS has a finer time frame, it only makes sense to extrapolate weekly hours from daily hours. Also, common knowledge suggests that the daily variation of hours worked within a typical week is more salient than the weekly variation within a typical month, quarter, or year, at least for most people.

\footnotetext{
${ }^{4}$ The EFS became known as the Living Cost and Food Survey from January 2008.

${ }^{5}$ The CPS asks the respondents how many hours he/she usually works per week, and how many hours he/she actually worked the week before, both for their main jobs and other jobs.
} 
The rest of the paper is organized as follows. Section 2 gives more information about time use surveys and commonly used surveys, focusing on how hours worked are measured in the ATUS, the DTUS and the CPS. In Section 3, we first give two impossibility results regarding the true weekly hours. Then we focus on the estimation of labor supply parameters. We set up the model and propose several intuitive estimators which utilize the ATUS daily hours, and investigate their asymptotic properties such as consistency, asymptotic normality and asymptotic efficiency. We recommend the imputed estimator based on our analysis. Section 4 uses the DTUS data to show some distributional properties of the measurement error in the recalled hours worked and contains simulation experiments based on the DTUS data to illustrate the finite sample properties of different estimators. Section 5 provides our empirical study of the labor supply elasticities at the intensive margin. Section 6 concludes the paper.

\section{Time Use Surveys}

The ATUS randomly draws roughly a one-eighth subsample of the respondents who just completed their participation in the CPS within the past two to five months. On a randomly chosen day (survey day), the respondents are asked to fill up a diary detailing all their activities minute-by-minute on the previous day (diary day). Adding all the time spent working by each respondent yields his/her ATUS hours worked for the diary day. Since the respondents of the ATUS had already participated in the CPS, all the data collected by the ATUS and the CPS about them are available for analysis, including demographics and income.

The ATUS has some distinct features that set it apart from commonly used surveys like the CPS. First, the respondents of the ATUS recall their activities for only one day ago, as opposed to weeks or months. Second, the ATUS imposes a 24-hour limit on the time allocated to all the recalled activities. These two features are likely to make the ATUS hours a much more accurate measure of the hours worked on a single day. Throughout this paper, we assume that the observed daily hours worked in the ATUS are the true hours worked for the diary day, without any measurement error. We fully acknowledge that this assumption is almost certainly wrong, and that the incidence and the size of the measurement error in the ATUS daily hours should be carefully examined for any serious empirical research. But it is adequate and convenient for our purpose in this paper.

On the contrary, the CPS records weekly hours, by asking either how many hours the respondents usually work per week or how many hours they actually worked in the previous week. While probably less accurate than the ATUS hours, the CPS hours concern a longer time period. 
In order to quantify and rectify the consequences of error-ridden hours in the CPS using the more accurate ATUS hours, we have to understand and tackle this time specificity of the two data sources. As mentioned before, this time specificity is the crux of this paper.

Such time specificity of hours between time use surveys and commonly used surveys is not unique to the US, presumably because of high costs of conducting time use surveys. In fact, to the best of our knowledge, the only country that has ongoing time use survey that records activities for an entire week is the Netherlands. The DTUS has been carried out since 1975 and has been published every five years. In the week long diary, the participants record their main activity every ten minutes and a secondary activity that might take place at the same time. The survey randomly draw more than two thousand participants from the Dutch population aged 12 and over since 2006. Since the DTUS also contains CPStype recalled weekly hours, it serves as a particularly precious benchmark against which we can evaluate different estimators. We are going to base our simulation studies on the DTUS. Unfortunately, the DTUS does not contain detailed information on income, which renders it unsuitable for our empirical analysis involving wage or earnings. But the DTUS contains demographic information which allows us to draw some empirical findings about labor supply along that line.

\section{Good News and Bad News about Labor Supply}

This section has good news and bad news. We start with the bad news - that is, what time use surveys cannot tell us about labor supply. By a very simple and straightforward potential outcome argument, we show that neither the weekly number of hours worked nor its distribution can be identified using the ATUS type time use survey data. Then we proceed to the good news - that is, what time use surveys can tell us.

\subsection{Bad News: Potential Hours and Impossibility Results}

Now we formally present our analysis. Let the individual respondents be indexed by $i$, and $i \in\{1, \ldots, n\}$. Let $H_{i}^{w}$ denote the true weekly hours worked by individual $i$. The recalled weekly number of hours worked $H_{i}^{C P S}$ in the CPS is an error-ridden measure of $H_{i}^{w}$,

$$
H_{i}^{C P S}=H_{i}^{w}+e_{i}
$$

The measurement error is nonclassical implies that $e_{i}$ could be correlated with $H_{i}^{w}$.

\footnotetext{
${ }^{6}$ The Economic and Social Research Council (ESRC) of the UK funded time use survey in 1973, 1974, 1983 and 1984 that covers 7 days of a week, but the more recent time use survey in the UK covers only 2 days.
} 
Let $t \in\{1, \ldots, 7\}$ denote the days of a week, 7 and let $H_{i t}$ denote the true daily hours worked by individual $i$ on day $t$. Naturally, the weekly hours worked equal to the sum of daily hours worked over the week,

$$
H_{i}^{w}=\sum_{t=1}^{7} H_{i t}
$$

Let $t_{i}$ be the dairy day of individual $i$ in the ATUS, then the daily hours worked in ATUS, denoted as $H_{i}^{A T U S}$, is just $H_{i t_{i}}$. To facilitate our analysis, it helps to write the ATUS daily hours in an alternative way. Let $d_{i t} \equiv \mathbb{I}\left[t_{i}=t\right]$ be seven diary day dummy variables for each individual $i .8$ Then

$$
H_{i}^{A T U S}=H_{i t_{i}}=\sum_{t=1}^{7} d_{i t} H_{i t} .
$$

Since for any individual interviewed in the ATUS, one and only one of the seven diary dummies is one, we only have an accurate measure of his/her hours worked for a single day of the week. Therefore in general, it is impossible to recover individual weekly hours worked $H_{i}^{w}$ from what is available in the ATUS. In addition, finding out the distribution of $H_{i}^{w}$ requires joint distribution of $\left(H_{i 1}, \ldots, H_{i 7}\right)^{\prime}$, which ATUS has not information about. In consequence, even the distribution of the weekly hours worked $H_{i}^{w}$ cannot be identified using the ATUS daily hours data.

These impossibility results closely resembles the conventional wisdom in the program evaluation literature that even in purely random experiments, neither individual treatment effect nor its distribution in the population can be identified. 9 Following the convention, we call $H_{i t}$ "potential hours" of diary day $t(t=1, \ldots, 7)$.

The second impossibility result is best illustrated with the DTUS data. In Figure 1, the solid line shows the kernel density of DTUS weekly hours worked, which is directly observable in the DTUS for each individual. To mimic the ATUS, we randomly choose one day from the DTUS as the diary day for each individual, and plot the kernel density of the hours worked on the diary day multiplied by 7 . The dashed and the dotted lines in Figure 1 show the kernel densities for two such random experiments. The ATUS-type daily hours exhibit bimodal distributions as most people work very little hours on weekends, if at all. Figure 2 shows the results of a similar experiment which takes the common five-day work schedule into account. We only keep those individuals whose diary days are the workdays, and then multiple their ATUS-type daily hours by 5. As is shown in Figure 2, even though

\footnotetext{
${ }^{7} t=1$ indicates Sunday, $t=2$ indicates Monday, and so on.

${ }^{8}$ The symbol $\equiv$ indicates that the quantity on the left side is defined as the expression on the right side.

${ }^{9}$ Let $Y_{i 1}, Y_{i 0}$ and $d_{i}$ denote the outcome if treated, the outcome if not treated and the treatment indicator for individual $i$, respectively, then the observed outcome is $Y=d_{i} Y_{i 1}+\left(1-d_{i}\right) Y_{i 0}$. It is well known that the individual treatment effect, defined as $Y_{i 1}-Y_{i 0}$, cannot be identified.
} 


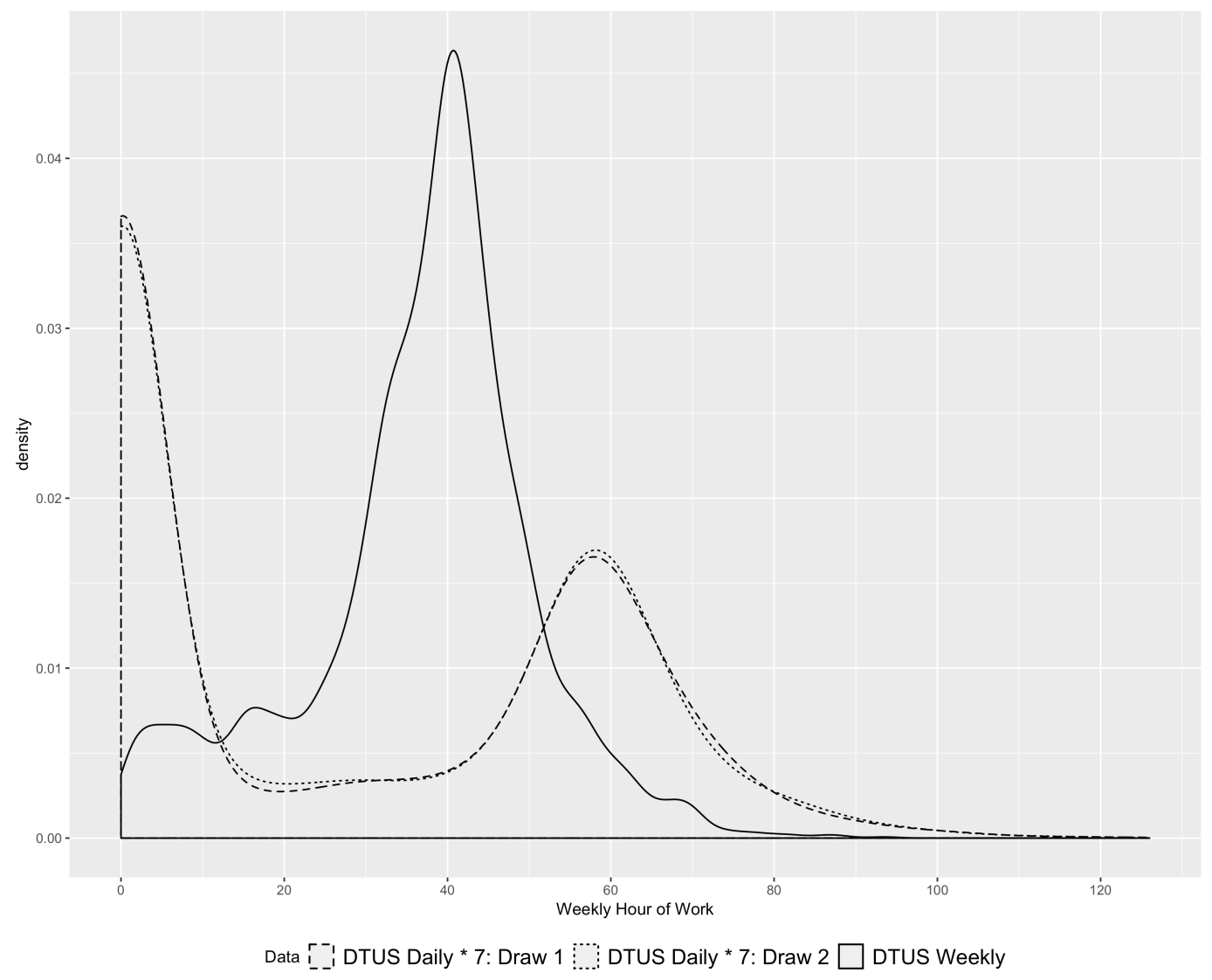

Figure 1: DTUS Weekly Hours vs. Randomly Drawn Daily Hours $\times 7$

the DTUS weekly hours and the scaled ATUS-type daily hours have similar mode, their distributions differ notably, especially toward the left end. The difference appears to be mainly driven by some people take a day off during the weekdays (and probably make up on the weekends.)

\subsection{Good News: Labor Supply Parameters}

Time use surveys speak volumes about weekly labor supply despite the above-mentioned impossibility results. We are interested in estimating the following linear model of weekly labor supply,

$$
H_{i}^{w}=X_{i}^{\prime} \beta+U_{i}, \quad i=1, \ldots, n,
$$

where $X_{i}$ is a $p \times 1$ vector of observable independent variables that affect hours worked with its first element being unit one. The explanatory variables $X_{i}$, including log wage in 


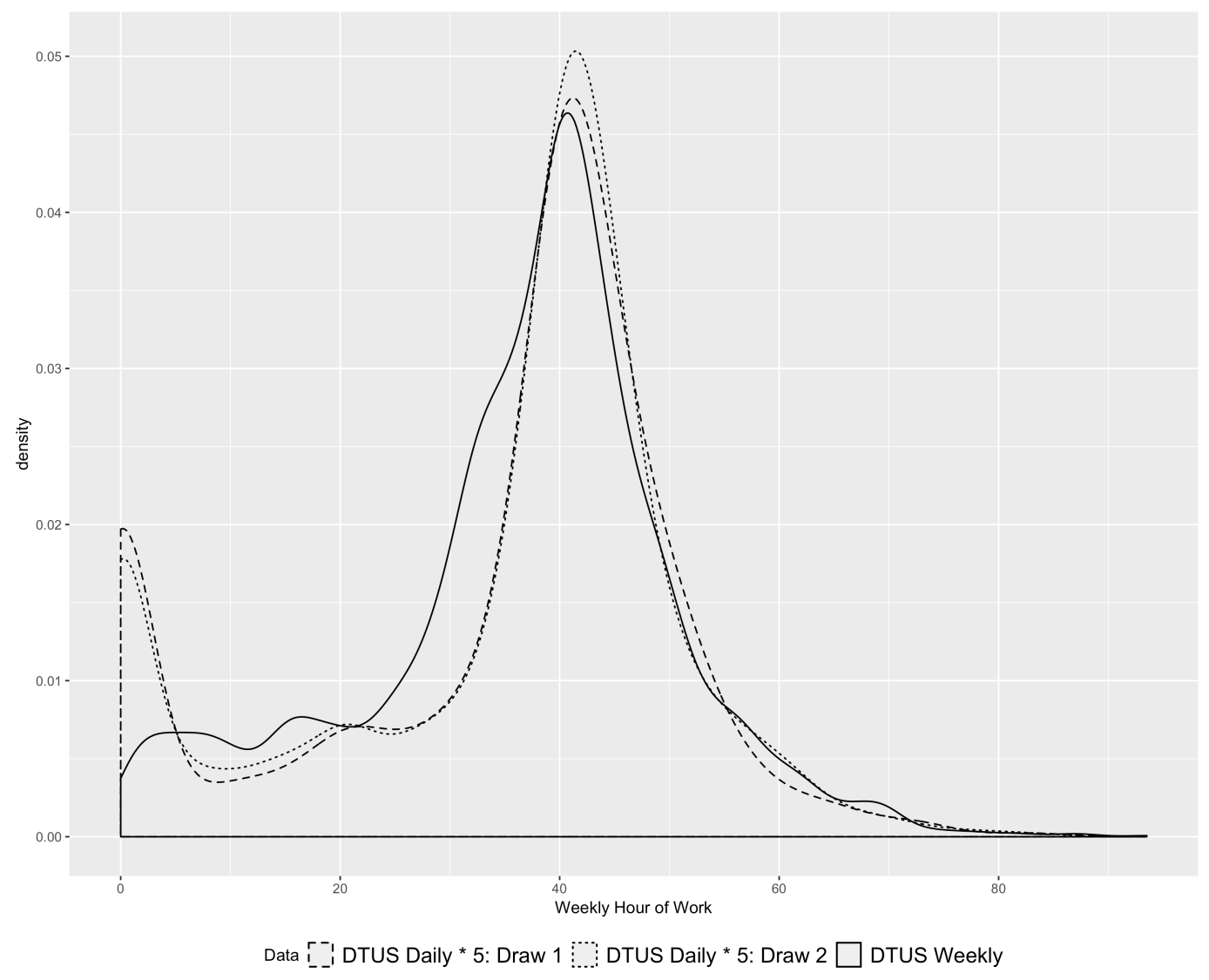

Figure 2: DTUS Weekly Hours vs. Randomly Drawn Weekday Daily Hours ×5

particular, tend to be correlated with $U_{i}$, and hence is often endogenous. For the purpose of this paper, we assume that a $q \times 1$ vector of IV $Z_{i}$ is available.10 Let $\sigma_{u}^{2} \equiv \operatorname{Var}\left(U_{i}\right)$.

The ideal case is when the true weekly hours worked $H_{i}^{w}$ were to be observable for each individual. The usual 2SLS estimator is then

$$
\hat{\beta}_{w k}=\left(X^{\prime} P_{z} X\right)^{-1}\left(X^{\prime} P_{z} H^{w}\right),
$$

where $H^{w} \equiv\left(H_{1}^{w}, \ldots, H_{n}^{w}\right)^{\prime}, X \equiv\left(X_{1}, \ldots, X_{n}\right)^{\prime}, Z \equiv\left(Z_{1}, \ldots, Z_{n}\right)^{\prime}$ and $P_{z} \equiv Z\left(Z^{\prime} Z\right)^{-1} Z^{\prime}$. Since it uses the unobservable true weekly hours worked, we call it week estimator. We maintain the following two assumptions throughout the paper.

Assumption 1 (Random sample). For any $i, j \in\{1, \ldots, n\}$ such that $i \neq j$,

$$
\left(H_{i}^{w}, H_{i 1}, \ldots, H_{i 7}, X_{i}^{\prime}, Z_{i}^{\prime}, d_{i 1}, \ldots, d_{i 7}\right)^{\prime} \Perp\left(H_{j}^{w}, H_{j 1}, \ldots, H_{j 7}, X_{j}^{\prime}, Z_{j}^{\prime}, d_{j 1}, \ldots, d_{j 7}\right)^{\prime},
$$

\footnotetext{
10 Angrist (1991) proposes the use of group classification variable as IV. In particular, Juhn and Murphy (1997) and Blau and Kahn (2007) use wage deciles as IV for wage.
} 
that is, the individuals in the sample are randomly drawn.

Assumption 2 (Valid instrumental variables). Assume that $\mathrm{E}\left(U_{i} Z_{i}\right)=0$, rank $\mathrm{E}\left(Z_{i} Z_{i}^{\prime}\right)=q$ $(q \geq p)$, and $\operatorname{rank} \mathrm{E}\left(Z_{i} X_{i}^{\prime}\right)=p$.

Under Assumption 1 and 2, it is a standard result that $\hat{\beta}_{w k}$ is consistent for $\beta$ and has asymptotically normal distribution. Unfortunately, $\hat{\beta}_{w k}$ is infeasible since $H_{i}^{w}$ is unobservable, but it will be our benchmark throughout this paper.

The 2SLS estimator based on the CPS weekly hours, on the other hand, is in general inconsistent. This is again a well known consequence of the nonclassical measurement error $e_{i}$ defined in eq. (11). 11

Now we consider how to utilize the daily hours in the ATUS. Because the ATUS diary is randomly chosen, we maintain an additional assumption throughout the paper.12

Assumption 3 (Random diary day). Diary day dummies $\left(d_{i 1}, \ldots, d_{i 7}\right)^{\prime}$ are independent from $\left(X_{i}^{\prime}, Z_{i}^{\prime}, U_{i}^{\prime}, H_{i}^{w}, H_{i 1}, \ldots, H_{i 7}\right)^{\prime}$.

Start with the infeasible week estimator $\hat{\beta}_{w k}$, we propose a feasible estimator which deviates this benchmark as little as possible. In light of eq. (2) and the definition of $P_{z}$, the infeasible 2SLS estimator $\hat{\beta}_{w k}$ can be re-written as

$$
\hat{\beta}_{w k}=\left(X^{\prime} P_{z} X\right)^{-1} X^{\prime} P_{z} \sum_{t=1}^{7} H_{t}=\left(X^{\prime} P_{z} X\right)^{-1} X^{\prime} P_{z} Z \sum_{t=1}^{7}\left(Z^{\prime} Z / n\right)^{-1}\left(Z^{\prime} H_{t} / n\right)
$$

where $H_{t} \equiv\left(H_{1 t}, \ldots, H_{n t}\right)$ for each $t \in\{1, \ldots, 7\}$. By the simple law of large numbers, we know that

$$
\left(Z^{\prime} Z / n\right)^{-1}\left(Z^{\prime} H_{t} / n\right) \stackrel{p .}{\longrightarrow}\left[\mathrm{E}\left(Z_{i} Z_{i}^{\prime}\right)\right]^{-1} \mathrm{E}\left(Z_{i} H_{i t}^{\prime}\right)
$$

By Assumption 3, we have

$$
\left[\mathrm{E}\left(Z_{i} Z_{i}^{\prime}\right)\right]^{-1} \mathrm{E}\left(Z_{i} H_{i t}^{\prime}\right)=\left[\mathrm{E}\left(Z_{i} Z_{i}^{\prime} \mid d_{i t}=1\right)\right]^{-1} \mathrm{E}\left(Z_{i} H_{i t}^{\prime} \mid d_{i t}=1\right)
$$

This indicates that we can use the subsample for diary day $t$, instead of the entire sample, to estimate this object. For each $t \in\{1, \ldots, 7\}$, suppose the subsample for diary day $t$ is $n_{t}$, and let $D_{t}$ denote an $n \times n$ diagonal matrix with elements $d_{i t}(i=1, \ldots, n)$. What $D_{t}$ does

\footnotetext{
${ }^{11}$ The probability limit of $\hat{\beta}_{w k}^{C P S} \equiv\left(X^{\prime} P_{z} X\right)^{-1}\left(X^{\prime} P_{z} H^{C P S}\right)$, the 2SLS estimator based on the CPS weekly hours, is $\left(\mathrm{E}\left(X_{i} Z_{i}^{\prime}\right)\left[\mathrm{E}\left(Z_{i} Z_{i}^{\prime}\right)\right]^{-1} \mathrm{E}\left(Z_{i} X_{i}^{\prime}\right)\right)^{-1} \mathrm{E}\left(X_{i} Z_{i}^{\prime}\right)\left[\mathrm{E}\left(Z_{i} Z_{i}^{\prime}\right)\right]^{-1} \mathrm{E}\left(Z_{i} e_{i}\right)$, which is in general not zero, since $\mathbb{E}\left(Z_{i} e_{i}\right) \neq 0$ for the nonclassical measurement error $e_{i}$.

${ }^{12}$ In fact, we checked the correlation between the index of the diary day and all the other variables we used in the ATUS and the CPS. It has a significant non-zero correlation only with the daily hours observed in the ATUS, which is natural since the ATUS daily hours are determined as $H_{i t_{i}}=\sum_{t=1}^{7} d_{i t} H_{i t}$.
} 
is just to select the subsample for diary day $t$. Replace the last part of $\hat{\beta}_{w k}$ by its diary day $t$ counterpart, we get a new estimator

$$
\hat{\beta}_{i m} \equiv\left(X^{\prime} P_{z} X\right)^{-1} X^{\prime} P_{z} Z \sum_{t=1}^{7}\left(Z^{\prime} D_{t} Z / n_{t}\right)^{-1}\left(Z^{\prime} D_{t} H_{t} / n_{t}\right) .
$$

In practice, this estimator is easy to compute using the ATUS data by the following steps:

1. Regress $X_{i}$ on $Z_{i}$ using the entire sample and take the fitted values $\hat{X}_{i}$;

2. Regress $H_{i}^{A T U S}$ (i.e. $H_{i t_{i}}$ ) on $Z_{i}$ using the subsample $d_{i t}=1$ to get $\hat{\gamma}_{t}$ (one for each diary day $t$ ), and impute the weekly hours worked by $\hat{H}_{i}^{w}=\sum_{t=1}^{7} Z_{i}^{\prime} \hat{\gamma}_{t}$ for the entire sample;

3. Regress $\hat{H}_{i}^{w}$ on $\hat{X}_{i}$ using the entire sample and get $\hat{\beta}_{i m}$.

Compared to the usual 2SLS estimator, this estimator adds one more simple step in the middle where the values of the unobservable weekly hours $H_{i}^{w}$ is imputed based on the instrumental variables. We call this estimator imputed estimator.

Apparently, the imputed estimator is not the only way of utilizing the ATUS daily hours data. Since the diary day is chosen randomly, it appears natural to regard the diary day as a random sample of a single day from a week, and expect the day-to-day variation of hours worked within a week to cancel out in large samples. This intuition leads to what we call day estimator. In practice, one would adjust the sampling probability of the diary days, 13 and then implement the IV (2SLS) estimator. Let $H^{A T U S}$ denote the $n \times 1$ vector of ATUS daily hours, and let $R$ denote an $n \times n$ diagonal matrix with elements $n / n_{t_{i}}(i=1, \ldots, n)$. Then the day estimator is defined as

$$
\hat{\beta}_{d a y}=\left(X^{\prime} P_{z} X\right)^{-1}\left(X^{\prime} P_{z} R H^{A T U S}\right)=\left(X^{\prime} P_{z} X\right)^{-1} X^{\prime} P_{z}\left(\sum_{t=1}^{7} r_{n t} D_{t} H_{t}\right),
$$

where $r_{n t} \equiv n / n_{t}$.

The last estimator we consider relies on the disaggregation of the weekly labor supply model into a number of daily labor supply models; that is, for $t=1, \ldots, 7$,

$$
H_{i t}=X_{i}^{\prime} \beta_{t}+U_{i t},
$$

where $\mathrm{E}\left(U_{i t}\right)=0$. Then the parameters $\beta$ in the weekly labor supply model can be re-written as

$$
\beta=\sum_{t=1}^{7} \beta_{t}
$$

\footnotetext{
${ }^{13}$ The ATUS samples Mondays to Fridays with probability of 0.10 each, and it samples Saturdays and Sundays with probability of 0.25 each. Weekends are oversampled since they are more informative about people's activities other than work.
} 
Therefore, it seems to be a logical attempt to estimate $\beta$ using what we call within estimator, defined as

$$
\hat{\beta}_{w n}=\sum_{t=1}^{7} \hat{\beta}_{t}=\sum_{t=1}^{7}\left(X^{\prime} P_{z t} X\right)^{-1} X^{\prime} P_{z t} H_{t},
$$

where $\hat{\beta}_{t}(t=1, \ldots, 7)$ is the usual 2SLS estimator of $\beta_{t}$ using only the subsample for diary day $t$ and $P_{z t}=\left(D_{t} Z\right)\left(Z^{\prime} D_{t} Z\right)^{-1}\left(D_{t} Z\right)^{\prime}$.

Remark 1 (Exogenous $X_{i}$ ). If $X_{i}$ are exogenous (hence $X_{i}$ are their own IV), then it is easy to verify that $\hat{\beta}_{w n}$ is numerically identical to the imputed estimator $\hat{\beta}_{i m}$. The two differ if $X_{i}$ are endogenous.

Remark 2 (Log-log specification). One may also consider the following log hours specification,

$$
\left(\sum_{t=1}^{7} \ln H_{i t}\right)=X_{i}^{\prime} \beta+U_{i} .
$$

Note that $\sum_{t=1}^{7} \ln H_{i t}=7 \ln \bar{H}^{g}$, where $\bar{H}^{g}=\left(H_{i 1} \cdots H_{i 7}\right)^{1 / 7}$ is the geometric mean of the number of hours worked during a week. Our proposed estimators obviously can accommodate this specification.

Remark 3 (Measurement error in the ATUS hours). Let $H_{i t}$ be the true hours worked on day $t$, and let $H_{i t}^{A T U S}=H_{i t}+e_{i t}^{A T U S}$ be the ATUS hours if respondent $i$ was interviewed for his/her hours worked on day $t$. Here $e_{i t}^{A T U S}$ is the measurement error of the ATUS hours. When the diary day assignment is independent of the measurement error in addition to Assumption 3, and $e_{i t}^{A T U S}$ is uncorrelated with the IV $Z_{i}$, we still have

$$
\left[\mathrm{E}\left(Z_{i} Z_{i}^{\prime}\right)\right]^{-1} \mathrm{E}\left(Z_{i} H_{i t}^{\prime}\right)=\left[\mathrm{E}\left(Z_{i} Z_{i}^{\prime} \mid d_{i t}=1\right)\right]^{-1} \mathrm{E}\left(Z_{i} H_{i t}^{A T U S^{\prime}} \mid d_{i t}=1\right) .
$$

So the above arguments still follow.

\subsubsection{Large Sample Properties}

Theorem 1 (Consistency). Under Assumption 1 and S, we have that $\hat{\beta}_{w k}, \hat{\beta}_{i m}, \hat{\beta}_{\text {day }}$, and $\hat{\beta}_{w n}$ all converge to $\beta$ in probability as $n \rightarrow \infty$.

Remark 4 . We need to point out that all the estimators we consider, including the within estimator, are consistent under the relative weaker assumption that $\mathrm{E}\left(U_{i} Z_{i}\right)=0$, instead of stronger one that $\mathrm{E}\left(U_{i t} Z_{i}\right)=0$. That is, the IV only need to be valid for the weekly labor supply equation, and not necessarily so for the labor supply equations of each day. Even if each daily 2 SLS estimator $\hat{\beta}_{t}$ might not be consistent for $\beta_{t}$, the within estimator $\hat{\beta}_{w n}$ still is. 
To derive the asymptotic distributions of the estimators, it helps to consider the linear projection of $H_{i t}$ onto the space of $Z_{i}$ :

$$
H_{i t}=Z_{i}^{\prime} \alpha_{t}+V_{i t},
$$

and let $V_{t}=\left(V_{1 t}, \ldots, V_{n t}\right)^{\prime}$. By construction, $\mathrm{E}\left(V_{i t}\right)=0$ and $\mathrm{E}\left(Z_{i} V_{i t}\right)=0$.

Assumption 4 (Diary day sampling probability). Assume that each day of a week has a positive probability of being sampled. That is, $0<\operatorname{Pr}\left(d_{i t}=1\right)<1$ for each day $t \in\{1, \ldots, 7\}$.

Define $A \equiv B C B^{\prime}$ with $B \equiv \mathrm{E}\left(X_{i} Z_{i}^{\prime}\right)$ and $C \equiv \mathrm{E}\left(Z_{i} Z_{i}^{\prime}\right)$, and let $r_{t}=1 / \operatorname{Pr}\left(d_{i t}=1\right)$. The following theorem gives the relative efficiency of the estimators.

Theorem 2 (Asymptotic Normality I). Under Assumptions 14, we have the following asymptotic normality results:

(i) $\sqrt{n}\left(\hat{\beta}_{w k}-\beta\right) \stackrel{d}{\rightarrow} \mathcal{N}\left(0, \Omega_{w k}\right)$, with

$$
\Omega_{w k} \equiv \sigma_{u}^{2} A^{-1}
$$

(ii) $\sqrt{n}\left(\hat{\beta}_{i m}-\beta\right) \stackrel{d}{\rightarrow} \mathcal{N}\left(0, \Omega_{i m}\right)$, with $\Omega_{i m}=\Omega_{w k}+\Omega_{i m-w k}$, where

$$
\Omega_{i m-w k} \equiv A^{-1} B C^{-1}\left[\sum_{t=1}^{7}\left(r_{t}-1\right) \mathrm{E}\left(Z_{i} V_{i t} V_{i t} Z_{i}^{\prime}\right)-2 \sum_{1 \leq t<\tau \leq 7} \mathrm{E}\left(Z_{i} V_{i t} V_{i \tau} Z_{i}^{\prime}\right)\right] C^{-1} B^{\prime} A^{-1} ;
$$

(iii) $\sqrt{n}\left(\hat{\beta}_{\text {day }}-\beta\right) \stackrel{d_{\dot{1}}}{\rightarrow} \mathcal{N}\left(0, \Omega_{\text {day }}\right)$, with $\Omega_{\text {day }}=\Omega_{\text {im }}+\Omega_{\text {day-im }}$, where

$$
\Omega_{\text {day-im }} \equiv A^{-1} B C^{-1}\left[\sum_{t=1}^{7}\left(r_{t}-1\right) \mathrm{E}\left(Z_{i} \alpha_{t}^{\prime} Z_{i} Z_{i}^{\prime} \alpha_{t} Z_{i}^{\prime}\right)-2 \sum_{1 \leq t<\tau \leq 7} \mathrm{E}\left(Z_{i} \alpha_{t}^{\prime} Z_{i} Z_{i}^{\prime} \alpha_{\tau}^{\prime} Z_{i}^{\prime}\right)\right] C^{-1} B^{\prime} A^{-1}
$$

As is clearly shown in the proof, both $\Omega_{i m-w k}$ and $\Omega_{d a y-i m}$ are variance-covariance matrices of some random vectors, so they are both positive definite. This implies that

$$
\Omega_{d a y} \geq \Omega_{i m} \geq \Omega_{w k}
$$

When $r_{t}=7$ for any $t$, and $\alpha_{1}=\cdots=\alpha_{7}, \Omega_{\text {day-im }}=0$, hence $\Omega_{i m}=\Omega_{d a y}$.

To get a rough idea of how large the efficiency loss of $\hat{\beta}_{i m}$ is compared to $\hat{\beta}_{w k}$, that is related to $\Omega_{i m-w k}$ in theorem 2. Suppose that for any $t, \tau \in\{1, \ldots, 7\}$ such that $t \neq \tau$, the projection residuals $V_{i t}$ from $H_{i t}=Z_{i}^{\prime} \alpha_{t}+V_{i t}$ satisfy the homoskedasticity assumption $\mathrm{E}\left(V_{i t}^{2} \mid Z_{i}\right)=\sigma_{v, t}^{2}$ and $\mathrm{E}\left(V_{i t} V_{i \tau} \mid Z_{i}\right)=\sigma_{v, t \tau}$. It can be shown that under this homoskedasticity assumption,

$$
\Omega_{i m-w k}=\left[\sum_{t=1}^{7}\left(r_{t}-1\right) \sigma_{v, t}^{2}-2 \sum_{1 \leq t<\tau \leq 7} \sigma_{v, t \tau}\right] A^{-1}
$$


Using the DTUS, letting $X_{i}=Z_{i}$ be the male dummy variable, age, age-squared, the number of children, the dummy for working in private sector, and the dummy for living in an metropolitan area, and letting $r_{1}=r_{7}=4$ and $r_{2}=\cdots=r_{5}=10$ as in the ATUS sampling scheme, we get the following estimates:

$$
\sigma_{u}^{2}=146.2, \quad \text { and } \quad \sum_{t=1}^{7} \sigma_{v, t}^{2}\left(r_{t}-1\right)-2 \sum_{1 \leq t<\tau \leq 7} \sigma_{v, t \tau}=409.1 .
$$

Hence the asymptotic variance of $\hat{\beta}_{w k}$ and $\hat{\beta}_{i m}$ are

$$
\operatorname{Var}\left(\hat{\beta}_{w k}\right)=n^{-1} 146.2 A^{-1} \quad \text { and } \quad \operatorname{Var}\left(\hat{\beta}_{i m}\right)=n^{-1}(146.2+409.1) A^{-1} .
$$

This implies that if the correlation relationship among the variables is the same as the DTUS, then compared to a time use survey that follows respondents' activities for an entire week which enables the use of the week estimator $\hat{\beta}_{w k}$, the number of respondents surveyed has to be roughly 3.8 times larger in order to get an imputed estimator $\hat{\beta}_{i m}$ with the same precision.

Even though Theorem 2 gives explicit formulae for the asymptotic variances and provides insights to the relative efficiency of the estimators, it is hardly useful in practice without further assumptions. The reason is that neither of $\Omega_{w k}, \Omega_{i m}$ or $\Omega_{d a y}$ is estimable without further assumptions. To see this, note that $\sigma_{u}^{2}$ and $\sigma_{v, t \tau}(1 \leq t<\tau \leq 7)$ require observing hours on different days $(t \neq \tau)$ for the same individual. The ATUS only provides information on one single day for each individual, and hence is inadequate in this regard. This problem is just another manifestation of the impossibility results discussed in Section 3.1.

This impossibility problem is, however, easy to solve, if we are willing to make a stronger assumption on the instrumental variables.

Assumption 5 (Instrumental variable in daily equations). Assume that

$$
\mathrm{E}\left(U_{i t} Z_{i}\right)=0, \quad \text { for all } t=1, \ldots, 7 \text {. }
$$

Here we do not provide the asymptotic distribution for the within estimator $\hat{\beta}_{w n}$. There are several reasons. First, as explained above, none of the asymptotic variance formulae given in Theorem 2 is of particular practical use since they are not estimable using the ATUS data under Assumption 1 - 4. They will be estimable, however, if Assumption 5 holds. Second, as we mentioned before, Assumption 2 only guarantees that the IV are valid for the weekly labor supply equation, but not necessarily in the daily ones. This means that $\hat{\beta}_{t}$ might be inconsistent for $\beta_{t}$ for some $t$. The asymptotic distribution of IV estimators, when the IV are invalid, is very complicated in general (Kiviet and Niemczyk, 2009). In the following, we derive the asymptotic distributions for all the estimators we consider if Assumption 5 is satisfied. 
To state the results, we consider the linear projection of $X_{i}$ onto the space of $Z_{i}$ :

$$
X_{i}=Z_{i}^{\prime} \lambda+\epsilon_{i}
$$

and we have $\mathrm{E}\left(\epsilon_{i t}\right)=0$ and $\mathrm{E}\left(Z_{i} \epsilon_{i}\right)=0$ by construction.

Theorem 3 (Asymptotic Normality II). Under Assumption 1 . 5 , we have the following asymptotic normality results:

(i) $\sqrt{n}\left(\hat{\beta}_{i m}-\beta\right) \stackrel{d}{\rightarrow} \mathcal{N}\left(0, \Omega_{i m}\right)$, with

$$
\Omega_{i m} \equiv \Omega_{i m, 1}+\Omega_{i m, 2}+2 \Omega_{i m, 3},
$$

where

$$
\begin{gathered}
\Omega_{i m, 1} \equiv A^{-1} B C^{-1}\left[\sum_{t=1}^{7}\left(r_{t}-1\right) \mathrm{E}\left(Z_{i} \beta_{t}^{\prime} \epsilon_{i} \epsilon_{i}^{\prime} \beta_{t} Z_{i}^{\prime}\right)-2 \sum_{1 \leq t<\tau \leq 7} \mathrm{E}\left(Z_{i} \beta_{t} \epsilon_{i}^{\prime} \epsilon_{i} \beta_{\tau} Z_{i}^{\prime}\right)\right] C^{-1} B^{\prime} A^{-1} \\
\Omega_{i m, 2} \equiv A^{-1} B C^{-1}\left[\sum_{t=1}^{7} r_{t} \mathrm{E}\left(U_{i t}^{2} Z_{i} Z_{i}^{\prime}\right)\right] C^{-1} B^{\prime} A^{-1} \\
\Omega_{i m, 3} \equiv A^{-1} B C^{-1}\left[\sum_{t=1}^{7}\left(r_{t}-1\right) \mathrm{E}\left(U_{i t} Z_{i} \beta_{t}^{\prime} \epsilon_{i} Z_{i}^{\prime}\right)-2 \sum_{1 \leq t<\tau \leq 7} \mathrm{E}\left(U_{i \tau} Z_{i} \beta_{t}^{\prime} \epsilon_{i} Z_{i}^{\prime}\right)\right] C^{-1} B^{\prime} A^{-1}
\end{gathered}
$$

(ii) $\sqrt{n}\left(\hat{\beta}_{d a y}-\beta\right) \stackrel{d}{\rightarrow} \mathcal{N}\left(0, \Omega_{d a y}\right)$, with $\Omega_{d a y} \equiv \Omega_{i m}+\Omega_{\text {day-im }}$, where $\Omega_{i m}$ is defined in eq. (16) and $\Omega_{\text {day-im }}$ is defined in eq. (14);

(iii) $\sqrt{n}\left(\hat{\beta}_{w n}-\beta\right) \stackrel{d_{\dot{ }}}{\rightarrow} \mathcal{N}\left(0, \Omega_{w n}\right)$, with

$$
\Omega_{w n} \equiv A^{-1} \sum_{t=1}^{7} r_{t} \sigma_{u, t}^{2}, \quad \text { and } \quad \sigma_{u, t}^{2} \equiv \mathrm{E}\left(U_{i t}^{2}\right)
$$

The asymptotic variance of the infeasible weekly estimator $\hat{\beta}_{w k}$ is still not estimable under the stronger assumption, since $U_{i}$, the error term in the weekly labor supply equation is still not observable. This is not much of a problem, because $\hat{\beta}_{w k}$ is merely a convenient conceptual device rather than a practical tool.

We suggest making inference based on Theorem 3 , since the asymptotic variance formulae here lead to easily computable formulae for the standard errors for $\hat{\beta}_{i m}, \hat{\beta}_{d a y}$ and $\hat{\beta}_{w n}$. Before giving the standard error formulae, we need some notation. Note that the coefficient $\lambda$ in the linear projection eq. (15) of $X_{i}$ on $Z_{i}$ can be estimated using the entire sample. Define

$$
\hat{X}_{i} \equiv Z_{i}^{\prime} \hat{\lambda}, \quad \text { and } \quad \hat{\epsilon}_{i} \equiv Z_{i}-\hat{X}_{i} .
$$


Let

$$
A_{n} \equiv \frac{1}{n} \sum_{i=1}^{n} \hat{X}_{i} \hat{X}_{i}^{\prime}, \quad B_{n} \equiv \frac{1}{n} \sum_{i=1}^{n} X_{i} Z_{i}^{\prime}, \quad \text { and } \quad C_{n} \equiv \frac{1}{n} \sum_{i=1}^{n} Z_{i} Z_{i}^{\prime} .
$$

Note that the coefficient $\alpha_{t}$ in the linear projection eq. (11) of $H_{i t}$ on $Z_{i}$ can be estimated using the subsample for diary day $t$. For each individual $i=1, \ldots, n$, let

$$
\hat{U}_{i t_{i}} \equiv H_{i t_{i}}-Z_{i} \hat{\beta}_{t_{i}} .
$$

Recall that $t_{i}$ denote the diary day for individual $i$ in the ATUS data. For each $t=1, \ldots, 7$, define

$$
\hat{\sigma}_{u, t}^{2} \equiv \frac{1}{n_{t}} \sum_{i: t_{i}=t} \hat{U}_{i t_{i}}^{2}, \quad \hat{Q}_{t}^{u z} \equiv \frac{1}{n_{t}} \sum_{i: t_{i}=t} \hat{U}_{i t_{i}}^{2} Z_{i} Z_{i}^{\prime}
$$

$\hat{Q}_{t}^{z \epsilon} \equiv \frac{1}{n} \sum_{i=1}^{n} Z_{i} \hat{\beta}_{t}^{\prime} \hat{\epsilon}_{i} \hat{\epsilon}_{i}^{\prime} \hat{\beta}_{t} Z_{i}^{\prime}, \quad \hat{Q}_{t}^{u \epsilon} \equiv \frac{1}{n_{t}} \sum_{i: t_{i}=t} \hat{U}_{i t_{i}} Z_{i} \hat{\beta}_{t}^{\prime} \hat{\epsilon}_{i} Z_{i}^{\prime}, \quad$ and $\quad \hat{Q}_{t}^{\alpha} \equiv \frac{1}{n} \sum_{i=1}^{n} Z_{i} \hat{\alpha}_{t}^{\prime} Z_{i} Z_{i}^{\prime} \hat{\alpha}_{t} Z_{i}^{\prime}$.

And for each pair of $(t, \tau)$ such that $1 \leq t<\tau \leq 7$, define

$\hat{Q}_{t, \tau}^{z \epsilon} \equiv \frac{1}{n} \sum_{i=1}^{n} Z_{i} \hat{\beta}_{t}^{\prime} \hat{\epsilon}_{i} \hat{\epsilon}_{i}^{\prime} \hat{\beta}_{\tau} Z_{i}^{\prime}, \quad \hat{Q}_{t, \tau}^{u \epsilon} \equiv \frac{1}{n_{\tau}} \sum_{i: t_{i}=\tau} \hat{U}_{i t_{i}} Z_{i} \hat{\beta}_{t}^{\prime} \hat{\epsilon}_{i} Z_{i}^{\prime}, \quad$ and $\quad \hat{Q}_{t, \tau}^{\alpha} \equiv \frac{1}{n} \sum_{i=1}^{n} Z_{i} \hat{\alpha}_{t}^{\prime} Z_{i} Z_{i}^{\prime} \hat{\alpha}_{\tau} Z_{i}^{\prime}$.

Therefore, we can define

$$
\begin{gathered}
\hat{\Omega}_{i m, 1} \equiv A_{n}^{-1} B_{n} C_{n}^{-1}\left[\sum_{t=1}^{7}\left(r_{n t}-1\right) \hat{Q}_{t}^{z \epsilon}-2 \sum_{1 \leq t<\tau \leq 7} \hat{Q}_{t, \tau}^{z \epsilon}\right] C_{n}^{-1} B_{n}^{\prime} A_{n}^{-1}, \\
\hat{\Omega}_{i m, 2} \equiv A_{n}^{-1} B_{n} C_{n}^{-1}\left[\sum_{t=1}^{7} r_{n t} \hat{Q}_{t}^{u z}\right] C_{n}^{-1} B_{n}^{\prime} A_{n}^{-1}, \\
\hat{\Omega}_{i m, 3} \equiv A_{n}^{-1} B_{n} C_{n}^{-1}\left[\sum_{t=1}^{7}\left(r_{n t}-1\right) \hat{Q}_{t}^{u \epsilon}-2 \sum_{1 \leq t<\tau \leq 7} \hat{Q}_{t, \tau}^{u \epsilon}\right] C_{n}^{-1} B_{n}^{\prime} A_{n}^{-1}, \\
\hat{\Omega}_{\text {day-im }} \equiv A_{n}^{-1} B_{n} C_{n}^{-1}\left[\sum_{t=1}^{7}\left(r_{n t}-1\right) \hat{Q}_{t}^{\alpha}-2 \sum_{1 \leq t<\tau \leq 7} \hat{Q}_{t, \tau}^{\alpha}\right] C_{n}^{-1} B_{n}^{\prime} A_{n}^{-1},
\end{gathered}
$$

and

$$
\hat{\Omega}_{w n} \equiv A_{n}^{-1} \sum_{t=1}^{7} r_{n t} \hat{\sigma}_{u, t}^{2}
$$

Finally, define

$$
\hat{\Omega}_{i m}=\hat{\Omega}_{i m, 1}+\hat{\Omega}_{i m, 2}+\hat{\Omega}_{i m, 3}
$$

and

$$
\hat{\Omega}_{d a y}=\hat{\Omega}_{i m}+\Omega_{d a y-i m},
$$

Theorem 4 (Standard error). Under Assumption 1 - 5, we have the following results: (i) $\hat{\Omega}_{i m} \stackrel{p .}{\longrightarrow} \Omega_{i m} ;$ (ii) $\hat{\Omega}_{d a y} \stackrel{p .}{\longrightarrow} \Omega_{\text {day }} ;$ (iii) $\hat{\Omega}_{w n} \stackrel{p .}{\longrightarrow} \Omega_{w n}$. 


\section{Lessons from the Dutch Time Use Survey}

The respondents in the DTUS report their daily activities in an entire week. So we are able to directly observe the number of hours worked in one week from the time use survey. As mentioned before, we regard the DTUS diary hours as the true hours worked in that week for our purpose. For the same respondents, we can also observe their recalled weekly hours worked and some of their characteristics including age, gender, education and number of children, but we do not observe their earnings. 14 The recalled weekly hours worked in DTUS were generated by the following procedure. Respondents were asked to recall the number of hours worked during the week prior to the interview. The number of hours worked during the last week was given priority. If data on the number of hours worked last week was not available, then one uses the reported usual weekly hours worked. When neither question was available, the weekly hours worked from the 7-day diaries were used. Unfortunately, there is no variable that indicates which way was used to obtain one particular observation of recalled hours worked.

The sample we use in this section consists of individuals aged between 25 and 54 surveyed in 1980, 1985, 1990, 1995, 2000 and 2005, whose recalled hours and diary hours are both positive. The entire sample contains 6,567 individual-year records (Fisher et al., 2018).

\subsection{Simulations Based on the DTUS}

Based on the DTUS data, we design a simulation study to compare the finite sample performance of the estimators discussed previously. The nice thing about the DTUS is that it contains CPS-type recalled weekly hours, as well as ATUS-type daily hours for an entire week. As a result, we are able to compute the week estimator $\hat{\beta}_{w k}$ which would have been impossible for the ATUS.

Given the daily hours worked $H_{i t}^{D T U S}(t=1, \ldots, 7)$ in the DTUS, we generate a single endogenous regressor $\tilde{X}_{i}$ and a single instrumental variable $\tilde{Z}_{i}$ such that eq. (8) is satisfied with $X_{i}=\left(1, \tilde{X}_{i}\right)^{\prime}, Z_{i}=\left(1, \tilde{Z}_{i}\right)^{\prime}, \operatorname{Corr}\left(U_{i t} Z_{i}\right)=0$ for $t=1, \ldots, 7$. In particular, let $H^{D T U S}$ denote the $n \times 7$ matrix with elements $H_{i t}^{D T U S}$, and let $T_{1}, \ldots, T_{7}$ be the principal components of $H^{D T U S}$. We set $\tilde{Z}_{i}$ to be the first principal component of $H^{D T U S}$, i.e. $\tilde{Z}=T_{1}$. To introduce the endogeneity, we generate an $n \times 7$ matrix of independent random variables from $N(0,2), 15$ denoted by $V$. Then we set $H_{i t}=H_{i t}^{D T U S}$ and $\tilde{X}_{i}=\tilde{Z}_{i}+\rho \sum_{t=1}^{7} V_{i t}$ for $i=1, \ldots, n$ and $t=1, \ldots 7$. The true parameters $\beta_{t}$ are therefore just the weights in $H_{t}$ $(t=1, \ldots, 7)$ associated with the first principal component. The true value of $\beta$ in eq. (4)

\footnotetext{
${ }^{14}$ The income variable in the DTUS is the annual income in quartiles.

${ }^{15}$ Such that $\operatorname{Var}\left(U_{i}\right) \approx \operatorname{Var}\left(T_{1}\right)$ in the exogenous regressor case.
} 
is therefore 2.2694.16 By varying $\rho$, we vary $\operatorname{Corr}\left(\tilde{X}_{i}, U_{i}\right)$, the degree of endogeneity of the regressor $\tilde{X}_{i}$. When $\rho=0$, the regressor is exogenous, and we try other values of $\rho$ such that $\operatorname{Corr}\left(\tilde{X}_{i}, U_{i}\right) \in\{0.25,0.5,0.75\}$. Note that as $\rho$ increases, the strength of the IV also decreases. For the above values of $\rho, \operatorname{Corr}\left(\tilde{X}_{i} \tilde{Z}_{i}\right)$ equals $1,0.95,0.80$ and 0.43 , respectively.

To illustrate the finite sample performance of the estimators, we randomly draw a subsample of size $n$ and vary $n \in\{200,500,1000,5000\}$. For each individual in the simulated sample, we randomly draw one day as the diary day, with the same probabilities as the ATUS. 17 We repeat the experiment $R=1000$ times, and report the mean squared errors (MSE), squared biases and variances for all the estimators considered in Table 1 .

Some patterns are apparent. First, the usual 2SLS estimator using the CPS-type recalled weekly hours $\hat{\beta}_{C P S}$ has the largest MSE in almost all parameterizations, which is roughly ten times of the maximum among all the other estimators. The large MSE is nearly entirely driven by the large bias, which is in turn a result of nonclassical measurement error in the CPS-type recalled weekly hours. Below we will illustrate this nonclassical measurement error using the DTUS data in Figure 3. Second, for almost all parameterizations, the biases of all the estimators based on the diary hours are negligible, and the differences in the performance of $\hat{\beta}_{w k}, \hat{\beta}_{i m}, \hat{\beta}_{d a y}$ and $\hat{\beta}_{w n}$ reside in efficiency. Third, since the infeasible week estimator $\hat{\beta}_{w k}$ uses the diary of an entire week that is usually not available in time use surveys, it is much more efficient than the others. This verifies the result of Theorem 2. Fourth, the imputed estimator $\hat{\beta}_{i m}$ is more efficient than $\hat{\beta}_{d a y}$ and $\hat{\beta}_{w n}$ in all parameterizations. Again, this verifies the result of Theorem 2. Fifth, when the regressor is exogenous, $\hat{\beta}_{i m}$ and $\hat{\beta}_{w n}$ perform equally well. This is because, as we mentioned before, the two estimators are numerically the same in this case. Last but not least, the within estimator $\hat{\beta}_{w n}$ appears to be unstable, especially when the sample size is smaller and when the instrument is weaker. The reason is that $\hat{\beta}_{w n}$ relies on the daily 2SLS estimators $\hat{\beta}_{t}$. When the sample size is small, the effective sample size for each day gets even smaller, and taking the inverse of the sample average matrices magnifies the sampling errors substantially.

\subsection{Empirical Findings from the DTUS}

We now turn to the empirical implications of the DTUS. We show some distributional features of the measurement error in the recalled hours, the effects of the number of children on labor supply, and the serial correlation of daily number of hours worked.

Figure 3 shows some distributional features of the measurement error in the recalled weekly hours worked in the Dutch data. The "measurement error" in Figure 3 equals

\footnotetext{
${ }^{16} \beta_{1}=0.0007, \beta_{2}=0.4379, \beta_{3}=0.4554, \beta_{4}=4576, \beta_{5}=0.4528, \beta_{6}=0.4304$ and $\beta_{7}=0.0346$.

${ }^{17}$ That is, the probability of being drawn is 0.25 for $t=1$ (Sundays) and $t=7$ (Saturdays), and 0.1 for the others.
} 


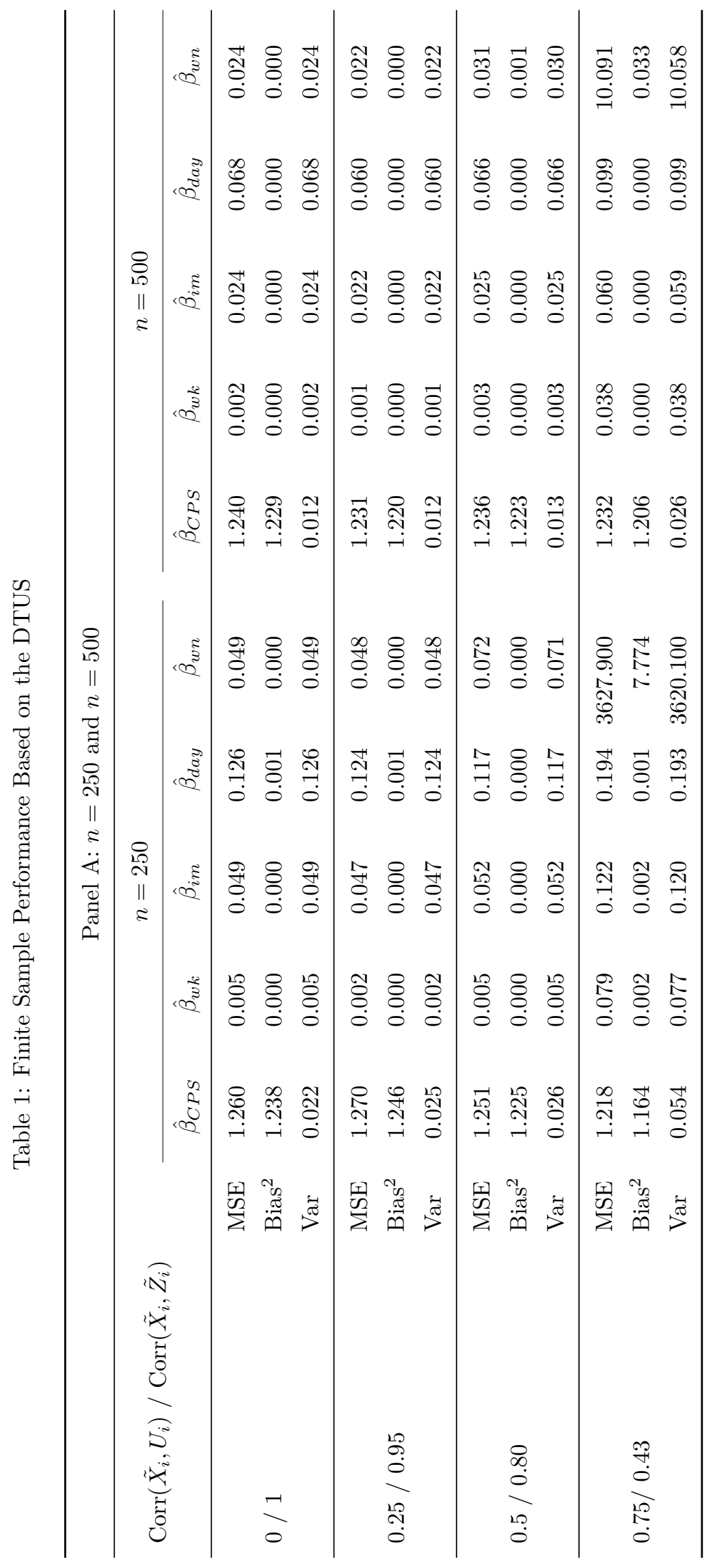




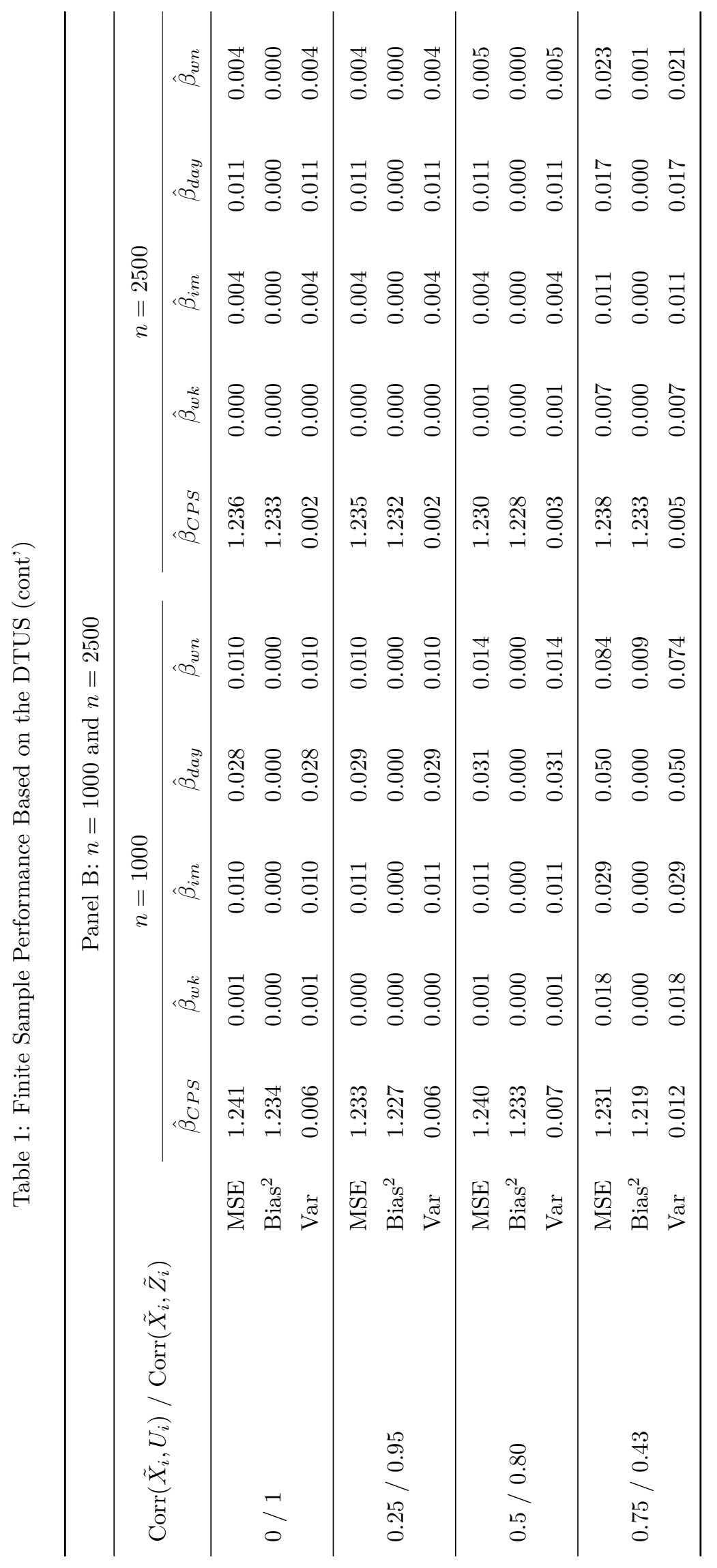



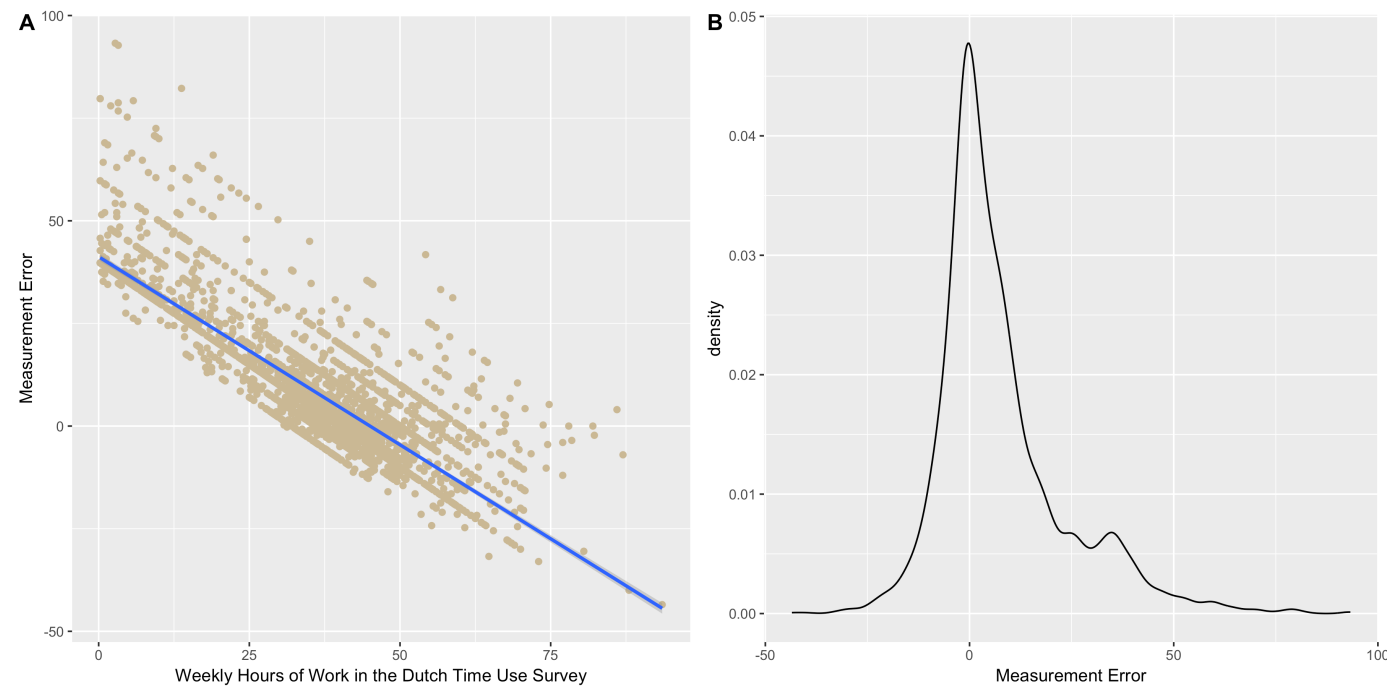

Figure 3: Kernel density of the measurement error in the recalled hours worked and its correlation with the weekly hours worked from DTUS

the recalled weekly hours worked minus the weekly hours worked from the 7-day diaries in DTUS. If the recalled number of hours worked do not have measurement error or the measurement error is classical, the recalled weekly hours worked minus the weekly hours worked from the 7-day diaries (that is the "measurement error" in Figure 3) is likely to be uncorrelated with the hours from the 7-day diaries. Panel A of Figure 3 suggests that the measurement error in recalled hours is nonclassical as it is negatively correlated with the hours from time use survey. Its kernel density (panel B) suggests that people are more likely to overstate the number of hours worked when they recall. The negative correlation between the measurement error and the true value of hours worked was first observed by Bound et al. (1989) in the PSID validation studies to the best of our knowledge.

Table 2 shows the effects of the number of children on labor supply. We run linear regression of weekly number of hours worked on the number of children under age 18, and other control variables. We used both the recalled weekly number of hours worked and the weekly number of hours worked from the time use survey as the dependent variable. The results from the two measures are considerably different. Using the hours worked from the time use survey, the number of children has big negative and significant effect on the hours worked for married women, but such effect is positive and insignificant when one used recalled number of hours worked. For married women, the time use survey implies that education has significant and positive effect on labor supply at the intensive margin, but using the recalled hours, one will conclude that education has insignificant effects. For 
Table 2: Labor Supply Estimation: DTUS

\begin{tabular}{|c|c|c|c|c|}
\hline & \multicolumn{2}{|c|}{ Married Men } & \multicolumn{2}{|c|}{ Married Women } \\
\hline & $\begin{array}{l}\text { Recalled } \\
\text { Hours }\end{array}$ & $\begin{array}{l}\text { Time Use } \\
\text { Hours }\end{array}$ & $\begin{array}{l}\text { Recalled } \\
\text { Hours }\end{array}$ & $\begin{array}{l}\text { Time Use } \\
\text { Hours }\end{array}$ \\
\hline Number of children age $<18$ & $\begin{array}{c}0.564^{* *} \\
(0.160)\end{array}$ & $\begin{array}{c}0.129 \\
(0.219)\end{array}$ & $\begin{array}{c}0.279 \\
(0.312)\end{array}$ & $\begin{array}{c}-4.328^{* *} \\
(0.396)\end{array}$ \\
\hline Education: completed 2ndry & $\begin{array}{c}0.901^{*} \\
(0.450)\end{array}$ & $\begin{array}{r}-0.144 \\
(0.616)\end{array}$ & $\begin{array}{l}-1 \\
(0.837)\end{array}$ & $\begin{array}{c}3.634^{* *} \\
(1.060)\end{array}$ \\
\hline Education: above 2ndry & $\begin{array}{l}1.907^{* *} \\
(0.474)\end{array}$ & $\begin{array}{r}-0.759 \\
(0.649)\end{array}$ & $\begin{array}{c}0.568 \\
(0.948)\end{array}$ & $\begin{array}{c}6.104^{* *} \\
(1.201)\end{array}$ \\
\hline
\end{tabular}

The other control variables are age, age-squared, a dummy of working in private sector (with public sector as base group), a urban area dummy (with rural being base group), and year dummies.

* denotes significance at $5 \%$, two-tailed test.

** denotes significance at $1 \%$, two-tailed test.

married men, we did not find significant effect of the number of children on labor supply, but such effect is significant and positive, though small in magnitude, from using recalled hours.

The daily hours worked are usually serially correlated. It is instructive to calculate the correlation matrix of daily hours worked within a week. Panel A of table 3 is the correlation matrix of daily hours worked. Reading the matrix, the daily hours worked on weekends are weakly correlated with hours worked on weekdays. The correlation coefficients between hours worked on weekdays are more or less the same - about 0.5. This suggests that the correlation between daily hours worked is likely due to the presence of workers' fixed effect. Panel B of the table is to see the correlation between hours after controlling workers' observed characteristics, including age, the number of children, industry, and gender. After controlling the observed characteristics, the correlation coefficients are still large. It suggests that the unobserved characteristics explain most of the correlation between daily hours worked. 
Table 3: Correlation matrix of daily hours worked

(a) Correlation matrix of daily hours worked

\begin{tabular}{rrrrrrrr}
\hline & Sun & Mon & Tue & Wed & Thu & Fri & Sat \\
\hline Sun & 1.00 & & & & & & \\
Mon & 0.04 & 1.00 & & & & & \\
Tue & 0.01 & 0.61 & 1.00 & & & & \\
Wed & 0.00 & 0.51 & 0.59 & 1.00 & & & \\
Thu & -0.01 & 0.48 & 0.54 & 0.59 & 1.00 & & \\
Fri & 0.04 & 0.41 & 0.46 & 0.48 & 0.56 & 1.00 & \\
Sat & 0.26 & 0.03 & 0.06 & 0.07 & 0.07 & 0.19 & 1.00 \\
\hline
\end{tabular}

(b) Correlation matrix of residuals from fitting daily hours worked by workers' characteristics ${ }^{1}$

\begin{tabular}{rrrrrrrr}
\hline & Sun & Mon & Tue & Wed & Thu & Fri & Sat \\
\hline Sun & 1.00 & & & & & & \\
Mon & 0.02 & 1.00 & & & & & \\
Tue & -0.00 & 0.52 & 1.00 & & & & \\
Wed & -0.02 & 0.38 & 0.48 & 1.00 & & & \\
Thu & -0.03 & 0.36 & 0.43 & 0.48 & 1.00 & & \\
Fri & 0.03 & 0.29 & 0.35 & 0.35 & 0.47 & 1.00 & \\
Sat & 0.26 & 0.01 & 0.05 & 0.04 & 0.05 & 0.17 & 1.00 \\
\hline
\end{tabular}

1 The residuals are from the linear regression of daily hours worked on age, age-squared, the number of individuals under 18 in the household, dummy variable for private sector (with public sector as the base group), a metropolitan area dummy, and a sex dummy. 
Table 4: Descriptive Statistics of Working Time and Wages

\begin{tabular}{lcccc}
\hline & Unmarried & Married & Unmarried & Married \\
\hline $\begin{array}{l}\text { Mean of CPS weekly hours worked } \\
\text { Std. dev. }\end{array}$ & 38.52 & 39.70 & 35.54 & 32.52 \\
& $(7.36)$ & $(6.22)$ & $(8.63)$ & $(10.45)$ \\
Mean of ATUS hours worked on & 4.76 & 4.73 & 4.18 & 3.55 \\
interview day & $(4.45)$ & $(4.57)$ & $(4.21)$ & $(4.01)$ \\
Std. dev. & & & & \\
& 9.84 & 9.61 & 9.67 & 9.26 \\
$\begin{array}{l}\text { Lower bound of std. dev. of weekly } \\
\text { hours worked from ATUS }\end{array}$ & & & & \\
& 18.62 & 21.80 & 16.53 & 18.65 \\
$\begin{array}{l}\text { Mean of Hourly Wage (2017 USD) } \\
n \text { of Obs }\end{array}$ & 3901 & 3975 & 5772 & 5644 \\
\hline
\end{tabular}

\section{Empirical Findings from the American Time Use Sur- vey}

\subsection{Sample}

The data are from ATUS 2003-2017 and the CPS for the same individuals in the ATUS (Hofferth et al., 2018). The sample used in our analysis consists of hourly paid workers between the age of 25 and 54, whose usual number of weekly hours worked at his/her main job reported in CPS is positive. So we study the labor supply elasticities of working men and women. The age restriction is to avoid the issues of schooling and retirement decisions. To limit the importance of measurement error in hourly wage, the hourly wage distribution was trimmed at percentiles 1 from below and 99 from above, respectively. After trimming, the hourly wage in 2017 USD within the sample ranges from $\$ 5.2 \$ 67.8$ for male workers and from $\$ 3.6$ to $\$ 63.1$ for female workers.

Table 1 provides the descriptive statistics about number of hours worked and real hourly wage rate. According to table 4 , men have slightly longer working hours than women regardless of marital status; married men have slightly more number of working hours than unmarried men, but married women work less than unmarried women; and for both genders, the married have higher hourly wage than the unmarried. The lower bound of standard deviation of the number of weekly hours worked from ATUS is calculated in the following 
way. It is reasonable to assume that the correlation between the number of hours worked by the same person in two days, $H_{i t}$ and $H_{i t^{\prime}}$, is non-negative. By $H_{i}^{w}=\sum_{t=1}^{7} H_{i t}$, we have

$$
\operatorname{Var}\left(H_{i}^{w}\right) \geq \sum_{t=1}^{7} \operatorname{Var}\left(H_{i t}\right),
$$

where $\operatorname{Var}\left(H_{i t}\right)$ can be readily estimated by the sample variance of hours worked on day $t$ in the ATUS. Note that excepting for married women, the standard deviation of CPS weekly hours worked is smaller than the lower bound of standard deviation from the ATUS. This observation (the distribution of labor hours from the time diaries has more variance than the hours from CPS type surveys) has been found in literature (Juster and Stafford, 1991). The explanation is usually the bunching of reported hours in CPS and other conventional survey.

\subsection{Elasticities of Hours Worked by Gender and Marital Status}

We estimate the hours elasticities using the following simple regression model,

$$
H_{i}^{w}=\beta_{0}+\beta_{1} \ln w_{i}+\beta_{2} y_{i}^{s p}+X_{i}^{\prime} \beta_{3}+U_{i},
$$

where $\ln w_{i}$ is the $\log$ of hourly wage, $y_{i}^{s p}$ is the usual weekly earnings of $i$ 's spouse $\left(y_{i}^{s p}=0\right.$ for unmarried worker), and $X_{i}$ is a vector of control variables, including age, age-squared, the number of own children age 4 or under, the number of own children age between 5 and 18, eight Census division dummies, a metropolitan area dummy, dummies for black non-Hispanic, other race non-Hispanic, and Hispanic (with white non-Hispanic as the base group), year dummies, dummies for occupation and industry, dummies for grade 12 , some college and college graduates (with less than grade 12 as base education category). Unfortunately, besides categorical family income, ATUS does not have information about the other income besides spouse weekly earnings. Blau and Kahn (2007) used CPS-March data to study labor supply of married women. The other income is mostly insignificant and numerically very small after controlling spouse wage rate. So here we control spouse wage rate but not other income. For hourly paid workers, ATUS directly asks them the hourly wage rate. We use wage and spouse weekly earnings deciles as IV for wage and spouse weekly earnings, respectively. We exclude salaried workers because their hourly wage is much harder to measure.18 This is a linear-log model, hence the wage elasticity of hours worked, $e\left(H_{i}^{w}\right)$, equals

$$
e\left(H_{i}^{w}\right)=\beta_{1} / H_{i}^{w} .
$$

\footnotetext{
${ }^{18}$ In a typical survey, the hourly wage for salaried workers is total earnings during a particular period divided by the number of hours worked in that period.
} 
Table 5: Labor Supply Elasticities by Gender and Marital Status

\begin{tabular}{llcc}
\hline \multirow{2}{*}{ Gender } & Marital Status & $\begin{array}{c}\text { CPS Recalled } \\
\text { Weekly Hours }\end{array}$ & $\begin{array}{c}\text { ATUS Imputed } \\
\text { Weekly Hours }\end{array}$ \\
\hline Male & Married & $0.056^{* *}$ & -0.008 \\
& & $(0.008)$ & $(0.046)$ \\
Male & Unmarried & $0.116^{* *}$ & 0.071 \\
& & $(0.009)$ & $(0.045)$ \\
Female & Married & $0.164^{* *}$ & $0.105^{* *}$ \\
& & $(0.011)$ & $(0.036)$ \\
Female & Unmarried & $0.119^{* *}$ & 0.064 \\
& & $(0.010)$ & $(0.038)$ \\
\hline
\end{tabular}

\footnotetext{
${ }^{1}$ The elasticities are evaluated at the respective mean hours worked in each data source.

${ }^{2}$ Standard errors are in parentheses. For "ATUS Imputed Weekly Hours", the reported standard errors are calculated using the formulas in theorem 4 .
}

The reported estimates of elasticities here are evaluated at the mean number of hours worked per week. We focus on the hours decision rather than the participation decision by including only the individuals who were working in the sample. The estimated elasticities are at the intensive margin.

Table 5 shows the estimation results from using different measure of labor supply $H_{i}^{w}$. The "CPS Recalled Weekly Hours" column was obtained by letting $H_{i}^{w}$ equal the recalled usual number of weekly hours worked in CPS. In the "ATUS Imputed Weekly hours", $H_{i}^{w}$ equals our imputed weekly hours $\hat{H}_{i}^{w}$ from ATUS. In other words, we estimated the regression model by the imputed estimator. The reported standard error of estimates in this column were obtained from using the formula in Theorem 4. Note that despite the different measurement of hours worked, the set of workers are identical across the two columns.

The salient difference between the CPS and our ATUS estimates is that using the same model specification and the same sample, our ATUS estimates show that the wage elasticities of hours worked of married women are substantially higher than the elasticities of unmarried women and men. The labor economics literature (Blundell and MaCurdy, 1999; McClelland and Mok, 2012) has shown that labor supply elasticities of married women are higher than the elasticities of men and unmarried women. Men and single women usually must work to support themselves, so their elasticities are expected to be low. Labor supply 
elasticities depend on the elasticities at the extensive (participation) and intensive (hours worked) margin. It is believed that married women have substantially higher participation elasticities than men, and this leads to observed higher elasticities of married women (Heckman, 1993). Table 5 is interesting because it shows that using ATUS data, married women have higher elasticities on the intensive margin than men and unmarried women. This conclusion from ATUS is different from the conclusion from CPS, which indicates that elasticities of hours worked of married women are similar to men and unmarried women. Our estimated elasticities of hours worked of married women is close to Blundell, Duncan, and Meghir (1998), where the estimated elasticity is 0.17, for UK working women.

It is also noticeable that the elasticities estimates from CPS are always higher than the estimates from ATUS, which is also observed by Barrett and Hamermesh (2017). They argue that this is likely to result from the measurement error in the recalled hours worked in the CPS. As we have shown $\hat{H}_{i}^{w}$ is a consistent estimate of $\mathrm{E}\left(H_{i}^{w} \mid Z_{i}\right)$. Here $Z_{i}$ include $\widehat{\ln w_{i}}$ (the predicted log of hourly wage in the first step of 2SLS IV estimation) and the other exogenous variables. Without measured error, $H_{i}^{w}$ from the CPS must satisfy the condition that $H_{i}^{w}-\mathrm{E}\left(H_{i}^{w} \mid Z_{i}\right)$ is mean independent of $Z_{i}$. In other words, we should expect that $H_{i}^{w}-\hat{H}_{i}^{w}$ is uncorrelated with $Z_{i}$. To visualize this, Figure 4 shows the scatter plots of $H_{i}^{w}-\hat{H}_{i}^{w}$ against $\ln w_{i}$ with linear regression fit. One can see that the difference is largely positively correlated with $\ln w_{i}$ excepting for married women. This could explain why the elasticities from CPS are higher than the elasticities from ATUS for all groups excepting for married women. Note that this positive correlation cannot be explained by bunching, the usual suspect of nonclassical measurement error in CPS, which is likely to result in negative correlation between the measurement error and the wage rate.

Table 6 displays more estimation results for married men and women using CPS and ATUS. There are several interesting differences between CPS and ATUS with imputed weekly hours worked. For married women, the estimates from CPS and ATUS with imputed hours are mostly similar besides the wage elasticity. The only noticeable difference is that the effects of the number of children age between 5 and 18 on the hours worked by married women-CPS implies bigger negative effect. For married men, CPS suggests that wife's earnings have negligible impact on husband's hours worked, with which our ATUS estimates disagree. Based on ATUS imputed hours, wife's earnings decreases men's working hours, though the impact is less than than the impact of husband's income on married women's hours. 


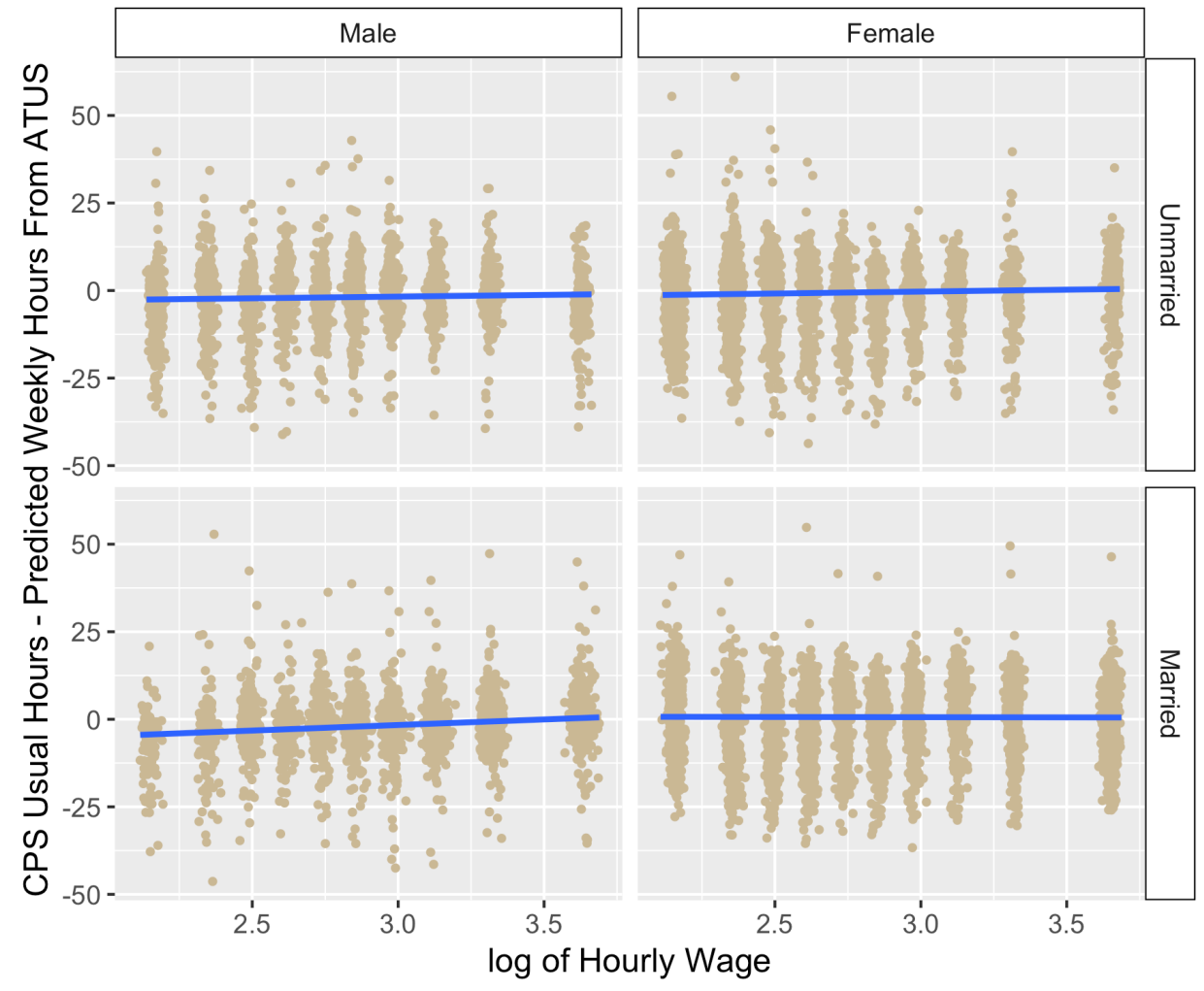

Figure 4: Difference Between Recalled and Imputed Weekly Hours Worked in CPS 


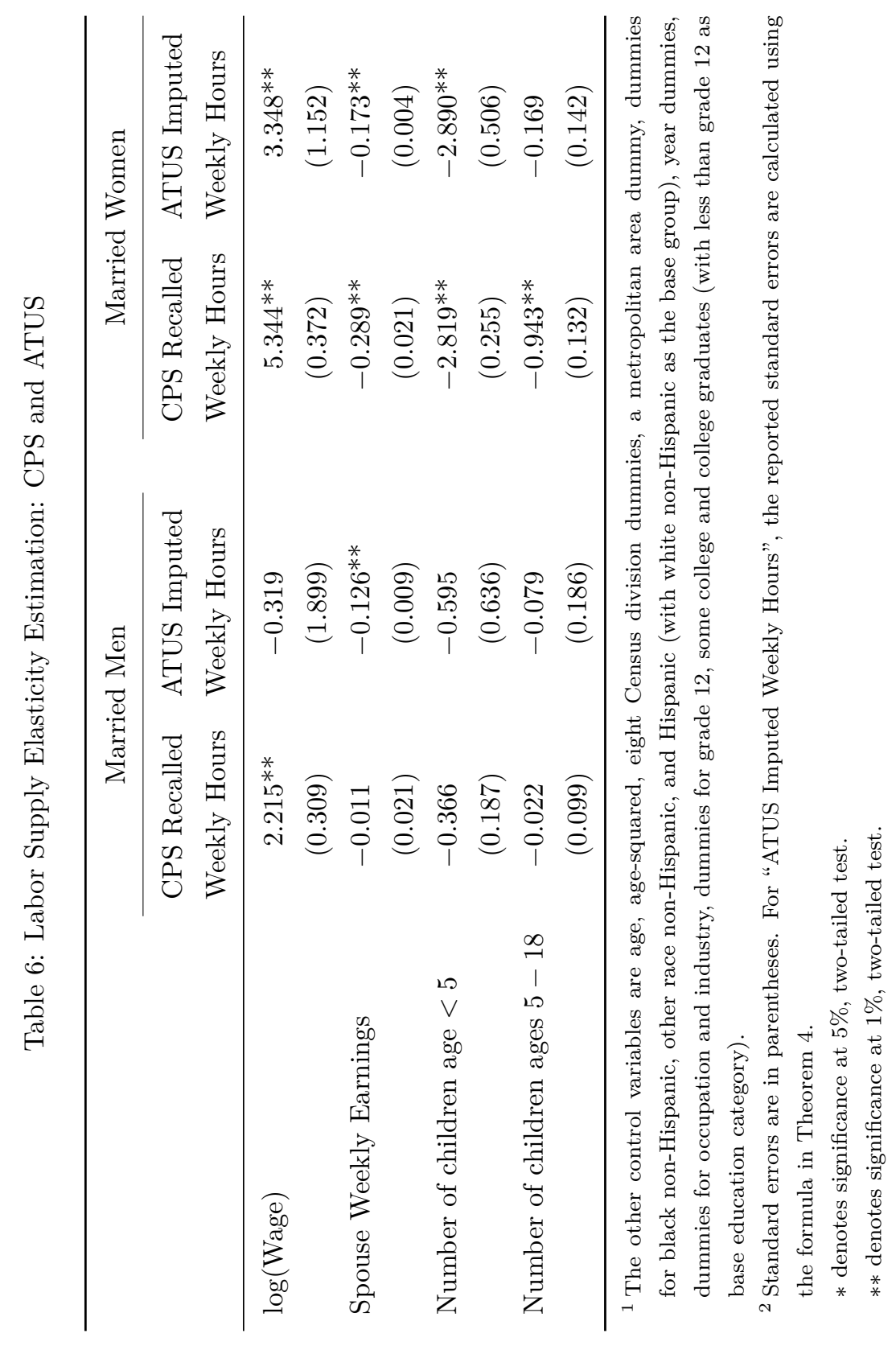




\section{Conclusion}

In this paper, we use the familiar potential outcome framework to demonstrate that weekly hours worked cannot be recovered from typical time use surveys. In spite of this impossibility, important parameters of labor supply can still be consistently and relatively efficiently estimated using time use surveys. We discuss the large sample properties of several intuitive estimators and recommend the imputed estimator on the ground of efficiency and robustness. The imputed estimator is a simple modification of the usual 2SLS estimator, which imputes the dependent variable as well as the independent variables using the instruments. We then proceed to illustrate the finite sample properties of all the estimators we consider in a simulation experiment based on the DTUS data, which tracks the respondents' activities for an entire week, and hence is a useful benchmark. Some empirical findings are also drawn from the DTUS data. Finally, we compare the estimated labor supply elasticities using the imputed estimator and that using the CPS recalled hours, and we are able to get a number of interesting empirical findings that are new in the labor economics literature. 


\section{Appendix}

\section{A Proofs}

Proof of Theorem 1. First, we show the consistency of $\hat{\beta}_{w k}$ :

$$
\hat{\beta}_{w k}-\beta=A_{n}^{-1} X^{\prime} P_{z} U=A_{n}^{-1} B_{n} C_{n}^{-1}\left(Z^{\prime} U / n\right) \stackrel{p .}{\longrightarrow} A^{-1} B C^{-1} \mathrm{E}\left(Z_{i} U_{i}\right)=0 .
$$

In fact, this is a standard result for instrumental variable estimators.

Second, we show the consistency of $\hat{\beta}_{i m}$. Consider the difference $\left(\hat{\beta}_{i m}-\hat{\beta}_{w k}\right)$ using their definitions:

$$
\begin{aligned}
\hat{\beta}_{i m}-\hat{\beta}_{w k} & =\left(X^{\prime} P_{z} X\right)^{-1} X^{\prime} P_{z}\left[\sum_{t=1}^{7} Z\left(Z^{\prime} D_{t} Z\right)^{-1} Z^{\prime} D_{t} H_{t}-H^{w}\right] \\
& =\left(X^{\prime} P_{z} X\right)^{-1} X^{\prime} P_{z}\left[\sum_{t=1}^{7} Z\left(Z^{\prime} D_{t} Z\right)^{-1} Z^{\prime} D_{t} H_{t}-P_{z} \sum_{t=1}^{7} H_{t}\right] \\
& =\sum_{t=1}^{7}\left(X^{\prime} P_{z} X\right)^{-1} X^{\prime} P_{z} Z\left[\left(Z^{\prime} D_{t} Z\right)^{-1} Z^{\prime} D_{t} H_{t}-\left(Z^{\prime} Z\right)^{-1} Z^{\prime} H_{t}\right] \\
& =\sum_{t=1}^{7}\left(X^{\prime} P_{z} X\right)^{-1} X^{\prime} Z\left[\left(Z^{\prime} D_{t} Z\right)^{-1} Z^{\prime} D_{t} H_{t}-\left(Z^{\prime} Z\right)^{-1} Z^{\prime} H_{t}\right] .
\end{aligned}
$$

Using the linear projection eq. (11), we have

$$
\hat{\beta}_{i m}-\hat{\beta}_{w k}=\sum_{t=1}^{7} A_{n}^{-1} B_{n}\left[\left(\frac{1}{n_{t}} Z^{\prime} D_{t} Z\right)^{-1} \frac{1}{n_{t}} Z^{\prime} D_{t} V_{t}-\left(\frac{1}{n} Z^{\prime} Z\right)^{-1} \frac{1}{n} Z^{\prime} V_{t}\right] .
$$

Define

$$
C_{n_{t}}=Z^{\prime} D_{t} Z / n_{t}
$$

Following from the law of large numbers, $A, B$ and $C$ are the probability limit of $A_{n}, B_{n}$, and $C_{n}$ (also $C_{n_{t}}$ ) as $n \rightarrow \infty$, respectively. By the definition of $A_{n}, B_{n}, C_{n}$ and $C_{n_{t}}$, we have

$$
\begin{aligned}
\hat{\beta}_{i m}-\hat{\beta}_{w k} & =\sum_{t=1}^{7} A_{n}^{-1} B_{n}\left[C_{n_{t}}^{-1} \frac{1}{n_{t}} Z^{\prime} D_{t} V_{t}-C_{n}^{-1} \frac{1}{n} Z^{\prime} V_{t}\right] \\
& \stackrel{p .}{\longrightarrow} \sum_{t=1}^{7} A^{-1} B C^{-1}\left[\mathrm{E}\left(Z_{i} d_{i t} V_{i t}\right)-\mathrm{E}\left(Z_{i} V_{i t}\right)\right] \\
& =\sum_{t=1}^{7} A^{-1} B C^{-1}\left[\mathrm{E}\left(Z_{i} V_{i t}\right) \mathrm{E}\left(d_{i t}\right)-\mathrm{E}\left(Z_{i} V_{i t}\right)\right] \\
& =0
\end{aligned}
$$


because $\mathrm{E}\left(Z_{i} V_{i t}\right)=0$. Since $\hat{\beta}_{w k} \stackrel{p .}{\longrightarrow} \beta$ and $\hat{\beta}_{i m}-\hat{\beta}_{w k} \stackrel{p .}{\longrightarrow} 0$, we conclude that $\hat{\beta}_{i m} \stackrel{p .}{\longrightarrow} \beta$.

Third, we show the consistency of $\hat{\beta}_{d a y}$. By the definition of $A_{n}, B_{n}, C_{n}$ and $C_{n_{t}}$, we have

$$
\begin{aligned}
\hat{\beta}_{d a y}-\hat{\beta}_{w k} & =\sum_{t=1}^{7} A_{n}^{-1} B_{n} C_{n}^{-1} \frac{Z^{\prime}\left(r_{n t} D_{t}-I\right) H_{t}}{n} \\
& \stackrel{p .}{\longrightarrow} A^{-1} B C^{-1} \sum_{t=1}^{7} \frac{Z^{\prime}\left(r_{t} D_{t}-I\right) H_{t}}{n} \\
& \stackrel{p .}{\longrightarrow} A^{-1} B C^{-1} \sum_{t=1}^{7} \mathrm{E}\left(\left(r_{t} d_{i t}-1\right) Z_{i} H_{i t}\right) \\
& =A^{-1} B C^{-1} \sum_{t=1}^{7} \mathrm{E}\left(r_{t} d_{i t}-1\right) \mathrm{E}\left(Z_{i} H_{i t}\right) \\
& =0
\end{aligned}
$$

where the second line holds because $r_{n t} \stackrel{p .}{\longrightarrow} r_{t}$, and the last equality holds since $\mathrm{E}\left(r_{t} d_{i t}-1\right)=$ 0 . Combined with the result that $\hat{\beta}_{w k} \stackrel{p .}{\longrightarrow} \beta$, this implies that $\hat{\beta}_{d a y} \stackrel{p .}{\longrightarrow} \beta$.

Fourth, we show the consistency of $\hat{\beta}_{w n}$. The weekly labor supply equation in eq. (4) can be re-written as the sum of seven daily labor supply equations in eq. (8), with

$$
\beta=\sum_{t=1}^{7} \beta_{t} \quad \text { and } \quad U_{i}=\sum_{t=1}^{7} U_{i t} .
$$

We then can re-write the within estimator as

$$
\begin{aligned}
\hat{\beta}_{w n} & =\sum_{t=1}^{7}\left(X^{\prime} P_{z t} X\right)^{-1} X^{\prime} P_{z t} H_{t} \\
& =\sum_{t=1}^{7}\left(X^{\prime} P_{z t} X\right)^{-1} X^{\prime} P_{z t}\left(X \beta_{t}+U_{t}\right) \\
& =\sum_{t=1}^{7} \beta_{t}+\sum_{t=1}^{7}\left(X^{\prime} P_{z t} X\right)^{-1} X^{\prime} P_{z t} U_{t} \\
& =\beta+\sum_{t=1}^{7}\left(X^{\prime} P_{z t} X\right)^{-1} X^{\prime} P_{z t} U_{t} .
\end{aligned}
$$

Simply by the law of large numbers, continuous mapping theorem, and the definition of $P_{z t}$, 
we have

$$
\begin{aligned}
\hat{\beta}_{w n}-\beta & =\sum_{t=1}^{7}\left(X^{\prime} P_{z t} X\right)^{-1} X^{\prime} P_{z t} U_{t} \\
& =\sum_{t=1}^{7}\left(\frac{X^{\prime} P_{z t} X}{n_{t}}\right)^{-1} \frac{X^{\prime} D_{t} Z}{n_{t}}\left(\frac{Z^{\prime} D_{t} Z}{n_{t}}\right)^{-1} \frac{Z^{\prime} D_{t} U_{t}}{n_{t}} \\
& \stackrel{p .}{\longrightarrow} \sum_{t=1}^{7} A^{-1} B C^{-1} \mathrm{E}\left(Z_{i} U_{i t}\right) \\
& =A^{-1} B C^{-1} \mathrm{E}\left(Z_{i} \sum_{t=1}^{7} U_{i t}\right) \\
& =A^{-1} B C^{-1} \mathrm{E}\left(Z_{i} U_{i}\right) \\
& =0 .
\end{aligned}
$$

This completes the proof.

Proof of Theorem 2. (i) We have

$$
\sqrt{n}\left(\hat{\beta}_{w k}-\beta\right)=A^{-1} \frac{1}{\sqrt{n}} X^{\prime} P_{z} U+o_{p}(1),
$$

which is asymptotically normal with mean zero and variance

$$
\Omega_{w k}=\sigma_{u}^{2} A^{-1},
$$

where $\sigma_{u}^{2} \equiv \operatorname{Var}\left(U_{i}\right)$. This completes the proof of (i). Again, this is a standard result for instrumental variable estimators.

To show (ii), we consider the decomposition

$$
\sqrt{n}\left(\hat{\beta}_{i m}-\beta\right)=\sqrt{n}\left(\hat{\beta}_{i m}-\hat{\beta}_{w k}\right)+\sqrt{n}\left(\hat{\beta}_{w k}-\beta\right) .
$$

Since the asymptotic variance of $\sqrt{n}\left(\hat{\beta}_{w k}-\beta\right)$ is given by (i), the key to finding the asymptotic distribution of $\sqrt{n}\left(\hat{\beta}_{i m}-\beta\right)$ is therefore to compute the asymptotic variance of $\sqrt{n}\left(\hat{\beta}_{i m}-\hat{\beta}_{w k}\right)$ and $\sqrt{n}\left(\hat{\beta}_{w k}-\beta\right)$, as well as their asymptotic covariance. Recall that eq. (26) implies

$$
\begin{aligned}
\sqrt{n}\left(\hat{\beta}_{i m}-\hat{\beta}_{w k}\right) & =\sum_{t=1}^{7} A_{n}^{-1} B_{n} \sqrt{n}\left[\left(\frac{1}{n_{t}} Z^{\prime} D_{t} Z\right)^{-1} \frac{n}{n_{t}} \frac{1}{n} Z^{\prime} D_{t} V_{t}-\left(\frac{1}{n} Z^{\prime} Z\right)^{-1} \frac{1}{n} Z^{\prime} V_{t}\right] \\
& =\sum_{t=1}^{7} A_{n}^{-1} B_{n}\left[C_{n_{t}}^{-1} r_{n t} \frac{1}{\sqrt{n}} Z^{\prime} D_{t} V_{t}-C_{n}^{-1} \frac{1}{\sqrt{n}} Z^{\prime} V_{t}\right] .
\end{aligned}
$$

Because $n^{-1 / 2} Z^{\prime} D_{t} V_{t}=O_{p}(1)$ and $n^{-1 / 2} Z^{\prime} V_{t}=O_{p}(1)$, we have

$$
\sqrt{n}\left(\hat{\beta}_{i m}-\hat{\beta}_{w k}\right)=A^{-1} B C^{-1} \sum_{t=1}^{7} \frac{1}{\sqrt{n}} Z^{\prime}\left(r_{t} D_{t}-I_{n}\right) V_{t}+o_{p}(1) .
$$


The focus is then the asymptotic distribution of

$$
\sum_{t=1}^{7} \frac{1}{\sqrt{n}} Z^{\prime}\left(r_{t} D_{t}-I_{n}\right) V_{t}=\sum_{t=1}^{7} \frac{1}{\sqrt{n}} \sum_{i=1}^{n}\left(r_{t} d_{i t}-1\right) Z_{i} V_{i t} .
$$

Because $d_{i t} \Perp\left(Z, H_{t}\right)$ and $\mathrm{E}\left(r_{t} d_{i t}-1\right)=0$, we have that $\mathrm{E}\left[\left(r_{t} d_{i t}-1\right) Z_{i} V_{i t}\right]=0$. Moreover, we have

$$
\mathrm{E}\left[\left(r_{t} d_{i t}-1\right) Z_{i} V_{i t} V_{i \tau} Z_{i}^{\prime}\left(r_{\tau} d_{i \tau}-1\right)\right]=\mathrm{E}\left[\left(r_{t} d_{i t}-1\right)\left(r_{\tau} d_{i \tau}-1\right)\right] \mathrm{E}\left(Z_{i} V_{i t} V_{i \tau} Z_{i}^{\prime}\right)
$$

It can be shown that

$$
\mathrm{E}\left[\left(r_{t} d_{i t}-1\right)\left(r_{\tau} d_{i \tau}-1\right)\right]= \begin{cases}r_{t}-1, & t=\tau \\ -1, & t \neq \tau\end{cases}
$$

We hence have

$$
\operatorname{Var}\left(\left(r_{t} d_{i t}-1\right) Z_{i} V_{i t}\right)=\left(r_{t}-1\right) \mathrm{E}\left(Z_{i} V_{i t} V_{i t} Z_{i}^{\prime}\right)
$$

and for $t \neq \tau$,

$$
\operatorname{Cov}\left(\left(r_{t} d_{i t}-1\right) Z_{i} V_{i t},\left(r_{\tau} d_{i \tau}-1\right) Z_{i} V_{i \tau}\right)=-\mathrm{E}\left(Z_{i} V_{i t} V_{i \tau} Z_{i}^{\prime}\right) .
$$

From eq. (27), we conclude that $\sqrt{n}\left(\hat{\beta}_{i m}-\hat{\beta}_{w k}\right)$ is asymptotically normal with mean zero and variance

$$
\Omega_{i m-w k} \equiv A^{-1} B C^{-1}\left[\sum_{t=1}^{7}\left(r_{t}-1\right) \mathrm{E}\left(Z_{i} V_{i t} V_{i t} Z_{i}^{\prime}\right)-2 \sum_{1 \leq t<\tau \leq 7} \mathrm{E}\left(Z_{i} V_{i t} V_{i \tau} Z_{i}^{\prime}\right)\right] C^{-1} B^{\prime} A^{-1} ;
$$

We then proceed to compute the covariance between $\sqrt{n}\left(\hat{\beta}_{i m}-\hat{\beta}_{w k}\right)$ and $\sqrt{n}\left(\hat{\beta}_{w k}-\beta\right)$. Note that we have shown $\mathrm{E}\left[\sqrt{n}\left(\hat{\beta}_{i m}-\hat{\beta}_{w k}\right)\right]=o_{p}(1)$ and $\mathrm{E}\left[\sqrt{n}\left(\hat{\beta}_{w k}-\beta\right)\right]=o_{p}(1)$. In addition, we have

$$
\begin{aligned}
& \mathrm{E}\left[\sqrt{n}\left(\hat{\beta}_{i m}-\hat{\beta}_{w k}\right) \sqrt{n}\left(\hat{\beta}_{w k}-\beta\right)\right] \\
= & A^{-1} B C^{-1} \mathrm{E}\left[\sum_{t=1}^{7} n^{-1} Z^{\prime}\left(r_{t} D_{t}-I_{n}\right) V_{t} U^{\prime} P_{z} X\right] A^{-1}+o_{p}(1) \\
= & A^{-1} B C^{-1} \sum_{t=1}^{7} \mathrm{E}\left[n^{-1} Z^{\prime}\left(r_{t} D_{t}-I_{n}\right) V_{t} U^{\prime} P_{z} X\right] A^{-1}+o_{p}(1) \\
= & A^{-1} B C^{-1} \sum_{t=1}^{7} \mathrm{E}\left[n^{-1} Z^{\prime} \mathrm{E}\left[\left(r_{t} D_{t}-I_{n}\right) V_{t} U^{\prime} P_{z} X \mid Z\right]\right] A^{-1}+o_{p}(1) \\
= & A^{-1} B C^{-1} \sum_{t=1}^{7} \mathrm{E}\left[n^{-1} Z^{\prime} \mathrm{E}\left(r_{t} D_{t}-I_{n}\right) \mathrm{E}\left(V_{t} U^{\prime} P_{z} X \mid Z\right)\right] A^{-1}+o_{p}(1),
\end{aligned}
$$


where the last equality holds because the diary day is completely random, i.e. $d_{i t}$ (and hence $D_{t}$ ) is independent from everything else. This, combined with

$$
\mathrm{E}\left(r_{t} D_{t}-I_{n}\right)=0
$$

implies

$$
\mathrm{E}\left[\sqrt{n}\left(\hat{\beta}_{i m}-\hat{\beta}_{w k}\right) \sqrt{n}\left(\hat{\beta}_{w k}-\beta\right)\right]=o_{p}(1)
$$

As a result,

$$
\operatorname{Cov}\left(\sqrt{n}\left(\hat{\beta}_{i m}-\hat{\beta}_{w k}\right), \sqrt{n}\left(\hat{\beta}_{w k}-\beta\right)\right)=o_{p}(1) .
$$

We conclude that the asymptotic variance of the imputed estimator equals

$$
\Omega_{i m}=\Omega_{w k}+\Omega_{i m-w k},
$$

This completes the proof of (ii).

To show (iii), we follow similar steps as for (ii). We decompose

$$
\sqrt{n}\left(\hat{\beta}_{d a y}-\beta\right)=\sqrt{n}\left(\hat{\beta}_{d a y}-\hat{\beta}_{i m}\right)+\sqrt{n}\left(\hat{\beta}_{i m}-\beta\right),
$$

where we only need to find the asymptotic variance of $\sqrt{n}\left(\hat{\beta}_{d a y}-\hat{\beta}_{i m}\right)$ and the asymptotic covariance between the two terms. First, we have

$$
\begin{aligned}
\sqrt{n}\left(\hat{\beta}_{\text {day }}-\hat{\beta}_{i m}\right) & =\sqrt{n}\left(X^{\prime} P_{z} X\right)^{-1} X^{\prime} Z \sum_{t=1}^{7}\left[\left(Z^{\prime} Z\right)^{-1} r_{n t} Z^{\prime} D_{t} H_{t}-\left(Z^{\prime} D_{t} Z\right)^{-1} Z^{\prime} D_{t} H_{t}\right] \\
& =A_{n}^{-1} B_{n} \sum_{t=1}^{7}\left(C_{n}^{-1}-C_{n_{t}}^{-1}\right) \frac{1}{\sqrt{n}} r_{n t} Z^{\prime} D_{t} H_{t} .
\end{aligned}
$$

In light of the linear projection eq. (11) of $H_{t}$, we have

$$
\begin{aligned}
\sqrt{n}\left(\hat{\beta}_{d a y}-\hat{\beta}_{i m}\right) & =A_{n}^{-1} B_{n} \sum_{t=1}^{7}\left(C_{n}^{-1}-C_{n_{t}}^{-1}\right) \frac{1}{\sqrt{n}} r_{n t} Z^{\prime} D_{t}\left(Z \alpha_{t}+V_{t}\right) \\
& =A_{n}^{-1} B_{n} \sum_{t=1}^{7}\left(C_{n}^{-1}-C_{n_{t}}^{-1}\right) \frac{1}{\sqrt{n}} r_{n t} Z^{\prime} D_{t} Z \alpha_{t}+o_{p}(1) \\
& =A_{n}^{-1} B_{n} \sum_{t=1}^{7}\left(C_{n}^{-1} \frac{1}{\sqrt{n}} Z^{\prime} r_{n t} D_{t} Z \alpha_{t}-\sqrt{n} \alpha_{t}\right)+o_{p}(1) \\
& =A_{n}^{-1} B_{n} \sum_{t=1}^{7}\left(C_{n}^{-1} \frac{1}{\sqrt{n}} Z^{\prime} r_{n t} D_{t} Z \alpha_{t}-\sqrt{n} C_{n}^{-1} \frac{Z^{\prime} Z}{n} \alpha_{t}\right)+o_{p}(1) \\
& =A_{n}^{-1} B_{n} C_{n}^{-1} \sum_{t=1}^{7}\left(\frac{1}{\sqrt{n}} Z^{\prime} r_{n t} D_{t} Z \alpha_{t}-\frac{1}{\sqrt{n}} Z^{\prime} Z \alpha_{t}\right)+o_{p}(1) \\
& =A^{-1} B C^{-1} \sum_{t=1}^{7} \frac{1}{\sqrt{n}} Z^{\prime}\left(r_{t} D_{t}-I_{n}\right) Z \alpha_{t}+o_{p}(1)
\end{aligned}
$$


where the second equality holds since $C_{n}^{-1}-C_{n_{t}}^{-1}=o_{p}(1), n^{-1 / 2} r_{n t} Z^{\prime} D_{t} V_{t}=O_{p}(1)$, and $C_{n_{t}}^{-1} Z^{\prime} D_{t} Z / n_{t}=I_{n}$, and the last equality holds by the definition of $C_{n}$ and $C_{n_{t}}$. It follows straightforward that $\sqrt{n}\left(\hat{\beta}_{d a y}-\hat{\beta}_{i m}\right)$ is asymptotically normal with some asymptotic variance $\Omega_{d a y-i m}$. To calculate $\Omega_{d a y-i m}$, let

$$
\delta_{i t}=\left(r_{t} d_{i t}-1\right) Z_{i} \alpha_{t}^{\prime} Z_{i},
$$

and rewrite

$$
\sqrt{n}\left(\hat{\beta}_{d a y}-\hat{\beta}_{i m}\right)=A^{-1} B C^{-1} \sum_{t=1}^{7} \frac{1}{\sqrt{n}} \sum_{i=1}^{n} \delta_{i t}+o_{p}(1) .
$$

Using eq. (28), we can show that

$$
\operatorname{Var}\left(\delta_{i t}\right)=\left(r_{t}-1\right) \mathrm{E}\left(Z_{i} \alpha_{t}^{\prime} Z_{i} Z_{i}^{\prime} \alpha_{t} Z_{i}^{\prime}\right)
$$

and

$$
\operatorname{Cov}\left(\delta_{i t}, \delta_{i \tau}\right)=-\mathrm{E}\left(Z_{i} \alpha_{t}^{\prime} Z_{i} Z_{i}^{\prime} \alpha_{\tau}^{\prime} Z_{i}^{\prime}\right)
$$

As a result,

$\Omega_{\text {day-im }}=A^{-1} B C^{-1}\left[\sum_{t=1}^{7}\left(r_{t}-1\right) \mathrm{E}\left(Z_{i} \alpha_{t}^{\prime} Z_{i} Z_{i}^{\prime} \alpha_{t} Z_{i}^{\prime}\right)-2 \sum_{1 \leq t<\tau \leq 7} \mathrm{E}\left(Z_{i} \alpha_{t}^{\prime} Z_{i} Z_{i}^{\prime} \alpha_{\tau}^{\prime} Z_{i}^{\prime}\right)\right] C^{-1} B^{\prime} A^{-1}$.

Second, we consider the asymptotic covariance between $\sqrt{n}\left(\hat{\beta}_{d a y}-\hat{\beta}_{i m}\right)$ and $\sqrt{n}\left(\hat{\beta}_{i m}-\beta\right)$. By the definition of $V_{i \tau}$ in the linear projection eq. (11), $Z_{i}$ and $V_{i \tau}(\tau=1, \ldots, 7)$ are orthogonal with each other. This implies that for any $1 \leq t \leq \tau \leq 7$,

$$
\operatorname{Cov}\left(\left(r_{t} d_{i t}-1\right) Z_{i} \alpha_{t}^{\prime} Z_{i},\left(r_{\tau} d_{i \tau}-1\right) Z_{i} V_{i \tau}\right)=0 .
$$

This further implies that $\sqrt{n}\left(\hat{\beta}_{d a y}-\hat{\beta}_{i m}\right)$ and $\sqrt{n}\left(\hat{\beta}_{i m}-\hat{\beta}_{w k}\right)$ are asymptotically uncorrelated. Furthermore, using the same argument as in the proof of (ii), one can show that $\sqrt{n}\left(\hat{\beta}_{\text {day }}-\right.$ $\left.\hat{\beta}_{i m}\right)$ and $\sqrt{n}\left(\hat{\beta}_{w k}-\beta\right)$ are asymptotically uncorrelated. Together they imply that $\sqrt{n}\left(\hat{\beta}_{d a y}-\right.$ $\left.\hat{\beta}_{i m}\right)$ and $\sqrt{n}\left(\hat{\beta}_{i m}-\beta\right)$ are asymptotically uncorrelated.

To summarize, we have shown that the asymptotic variance of $\sqrt{n}\left(\hat{\beta}_{d a y}-\beta\right)$ equals to

$$
\Omega_{d a y}=\Omega_{d a y-i m}+\Omega_{i m} .
$$

Note that since $\Omega_{d a y}$ is positive definite, it implies that $\hat{\beta}_{i m}$ is asymptotically more efficient than $\hat{\beta}_{d a y}$. This completes the proof of (iii).

Proof of Theorem 3. We first prove (i). Plugging eq. (8), the daily labor supply equation into eq. (6), the expression of the imputed estimator, and using the notation of $A_{n}, B_{n}, C_{n}$ and $C_{n_{t}}$, we can write

$$
\sqrt{n}\left(\hat{\beta}_{i m}-\beta\right)=\sqrt{n}\left(T_{i m, 1}-\beta\right)+\sqrt{n} T_{i m, 2},
$$


where

$$
\begin{aligned}
T_{i m, 1} & =\sum_{t=1}^{7}\left(X^{\prime} P_{z} X\right)^{-1} X^{\prime} P_{z} Z\left(Z^{\prime} D_{t} Z\right)^{-1} Z^{\prime} D_{t} X \beta_{t} \\
& =\sum_{t=1}^{7}\left(X^{\prime} P_{z} X\right)^{-1} X^{\prime} Z\left(Z^{\prime} D_{t} Z\right)^{-1} Z^{\prime} D_{t} X \beta_{t} \\
& =A_{n}^{-1} B_{n} \sum_{t=1}^{7} C_{n_{t}}^{-1} Z^{\prime} D_{t} X \beta_{t} / n_{t}, \\
T_{i m, 2} & =\sum_{t=1}^{7}\left(X^{\prime} P_{z} X\right)^{-1} X^{\prime} P_{z} Z\left(Z^{\prime} D_{t} Z\right)^{-1} Z^{\prime} D_{t} U_{t} \\
& =\sum_{t=1}^{7}\left(X^{\prime} P_{z} X\right)^{-1} X^{\prime} Z\left(Z^{\prime} D_{t} Z\right)^{-1} Z^{\prime} D_{t} U_{t}, \\
& =A_{n}^{-1} B_{n} \sum_{t=1}^{7} C_{n_{t}}^{-1} Z^{\prime} D_{t} U_{t} / n_{t} .
\end{aligned}
$$

We then proceed in three steps: (a) find the asymptotic distribution of $\sqrt{n}\left(T_{i m, 1}-\beta\right)$; (b) find the asymptotic distribution of $\sqrt{n} T_{i m, 2}$; and (c) find the asymptotic covariance between the two.

Using the linear projection eq. (15) and letting $\epsilon=\left(\epsilon_{1}, \ldots, \epsilon_{n}\right)^{\prime}$, we can re-write

$$
\begin{aligned}
T_{i m, 1} & =A_{n}^{-1} B_{n} \sum_{t=1}^{7} C_{n_{t}}^{-1} Z^{\prime} D_{t} Z \lambda \beta_{t} / n_{t}+A_{n}^{-1} B_{n} \sum_{t=1}^{7} C_{n_{t}}^{-1} Z^{\prime} D_{t} \epsilon \beta_{t} / n_{t} \\
& =A_{n}^{-1} B_{n} \lambda \sum_{t=1}^{7} \beta_{t}+A_{n}^{-1} B_{n} \sum_{t=1}^{7} C_{n_{t}}^{-1} Z^{\prime} D_{t} \epsilon \beta_{t} / n_{t}
\end{aligned}
$$

Also re-write $\beta$ using this linear projection and the notation of $A_{n}, B_{n}$ and $C_{n}$, we get

$$
\begin{aligned}
\beta & =\sum_{t=1}^{7} \beta_{t}=\sum_{t=1}^{7}\left(X^{\prime} P_{z} X\right)^{-1} X^{\prime} Z\left(Z^{\prime} Z\right)^{-1} Z^{\prime} X \beta_{t} \\
& =\sum_{t=1}^{7}\left(X^{\prime} P_{z} X\right)^{-1} X^{\prime} Z\left(Z^{\prime} Z\right)^{-1} Z^{\prime} Z \lambda \beta_{t}+\sum_{t=1}^{7}\left(X^{\prime} P_{z} X\right)^{-1} X^{\prime} Z\left(Z^{\prime} Z\right)^{-1} Z^{\prime} \epsilon \beta_{t} \\
& =A_{n}^{-1} B_{n} \lambda \sum_{t=1}^{7} \beta_{t}+A_{n}^{-1} B_{n} \sum_{t=1}^{7} C_{n}^{-1} \frac{1}{n} Z^{\prime} \epsilon \beta_{t}
\end{aligned}
$$

It then follows that

$$
\sqrt{n}\left(T_{i m, 1}-\beta\right)=A_{n}^{-1} B_{n} \sum_{t=1}^{7}\left[C_{n_{t}}^{-1} r_{n t} \frac{1}{\sqrt{n}} Z^{\prime} D_{t} \epsilon \beta_{t}-C_{n}^{-1} \frac{1}{\sqrt{n}} Z^{\prime} \epsilon \beta_{t}\right] .
$$


Because both $n^{-1 / 2} Z^{\prime} D_{t} \epsilon$ and $n^{-1 / 2} Z^{\prime} \epsilon$ are $O_{p}(1)$, we have

$$
\begin{aligned}
\sqrt{n}\left(T_{i m, 1}-\beta\right) & =A^{-1} B C^{-1} \sum_{t=1}^{7}\left[\frac{1}{\sqrt{n}} Z^{\prime}\left(r_{t} D_{t}-I_{n}\right) \epsilon\right] \beta_{t}+o_{p}(1) \\
& =A^{-1} B C^{-1} \sum_{t=1}^{7}\left[\frac{1}{\sqrt{n}} \sum_{i=1}^{n}\left(r_{t} d_{i t}-1\right) Z_{i} \epsilon_{i}^{\prime}\right] \beta_{t}+o_{p}(1) .
\end{aligned}
$$

Let

$$
\eta_{i t}=\left(r_{t} d_{i t}-1\right) Z_{i} \epsilon_{i}^{\prime} \beta_{t}
$$

so that we can write

$$
\sqrt{n}\left(T_{i m, 1}-\beta\right)=A^{-1} B C^{-1} \sum_{t=1}^{7} \frac{1}{\sqrt{n}} \sum_{i=1}^{n} \eta_{i t}+o_{p}(1) .
$$

Using eq. (28), we can show that

$$
\operatorname{Var}\left(\eta_{i t}\right)=\left(r_{t}-1\right) \mathrm{E}\left(Z_{i} \beta_{t}^{\prime} \epsilon_{i} \epsilon_{i}^{\prime} \beta_{t} Z_{i}^{\prime}\right)
$$

and

$$
\operatorname{Cov}\left(\eta_{i t}, \eta_{i \tau}\right)=-\mathrm{E}\left(Z_{i} \beta_{t} \epsilon_{i}^{\prime} \epsilon_{i} \beta_{\tau} Z_{i}^{\prime}\right)
$$

As a result, $\sqrt{n}\left(T_{i m, 1}-\beta\right)$ is asymptotically normal, and its asymptotic variance is

$$
\Omega_{i m, 1}=A^{-1} B C^{-1}\left[\sum_{t=1}^{7}\left(r_{t}-1\right) \mathrm{E}\left(Z_{i} \beta_{t}^{\prime} \epsilon_{i} \epsilon_{i}^{\prime} \beta_{t} Z_{i}^{\prime}\right)-2 \sum_{1 \leq t<\tau \leq 7} \mathrm{E}\left(Z_{i} \beta_{t} \epsilon_{i}^{\prime} \epsilon_{i} \beta_{\tau} Z_{i}^{\prime}\right)\right] C^{-1} B^{\prime} A^{-1} .
$$

Second, we consider the asymptotic distribution of $\sqrt{n} T_{i m, 2}$. Let

$$
\xi_{i t}=r_{t} d_{i t} Z_{i} U_{i t}
$$

Using the notation of $A_{n}, B_{n}, C_{n}$ and $C_{n_{t}}$, and because $n^{-1 / 2} Z^{\prime} D_{t} U_{t}=O_{p}(1)$, we can write

$$
\sqrt{n} T_{i m, 2}=A^{-1} B C^{-1} \sum_{t=1}^{7} \frac{1}{\sqrt{n}} \sum_{i=1}^{n} \xi_{i t}+o_{p}(1) .
$$

Note that we have

$\mathrm{E}\left(\xi_{i t}\right)=\mathrm{E}\left(r_{t} d_{i t}\right) \mathrm{E}\left(Z_{i} U_{i t}\right)=0, \quad \operatorname{Var}\left(\xi_{i t}\right)=r_{t} \mathrm{E}\left(U_{i t}^{2} Z_{i} Z_{i}^{\prime}\right), \quad$ and $\quad \operatorname{Cov}\left(\xi_{i t}, \xi_{i \tau}\right)=0$, since $d_{i t} d_{i \tau}=0$ for any $t \neq \tau$. Hence $\sqrt{n} T_{i m, 2}$ is asymptotically normal and its asymptotic variance is

$$
\Omega_{i m, 2}=A^{-1} B C^{-1}\left[\sum_{t=1}^{7} r_{t} \mathrm{E}\left(U_{i t}^{2} Z_{i} Z_{i}^{\prime}\right)\right] C^{-1} B^{\prime} A^{-1}
$$


Finally we consider the covariance between $\sqrt{n}\left(T_{i m, 1}-\beta\right)$ and $\sqrt{n} T_{i m, 2}$. We note that

$$
\mathrm{E}\left(\eta_{i t} \xi_{i \tau}^{\prime}\right)= \begin{cases}\left(r_{t}-1\right) \mathrm{E}\left(U_{i t} Z_{i} \beta_{t}^{\prime} \epsilon_{i} Z_{i}^{\prime}\right), & t=\tau \\ -\mathrm{E}\left(U_{i \tau} Z_{i} \beta_{t}^{\prime} \epsilon_{i} Z_{i}^{\prime}\right), & t \neq \tau\end{cases}
$$

Note that $\mathrm{E}\left(U_{i t} Z_{i} \beta_{t}^{\prime} \epsilon_{i} Z_{i}^{\prime}\right) \neq 0$ when $X_{i}$ is endogenous, so the covariance between $\sqrt{n}\left(T_{i m, 1}-\right.$ $\beta)$ and $\sqrt{n} T_{i m, 2}$ is

$$
\Omega_{i m, 3}=A^{-1} B C^{-1}\left[\sum_{t=1}^{7}\left(r_{t}-1\right) \mathrm{E}\left(U_{i t} Z_{i} \beta_{t}^{\prime} \epsilon_{i} Z_{i}^{\prime}\right)-2 \sum_{1 \leq t<\tau \leq 7} \mathrm{E}\left(U_{i \tau} Z_{i} \beta_{t}^{\prime} \epsilon_{i} Z_{i}^{\prime}\right)\right] C^{-1} B^{\prime} A^{-1} .
$$

We conclude that the asymptotic variance under the condition $\mathrm{E}\left(Z_{i} U_{i t}\right)=0$ for all $t$ is

$$
\Omega_{i m}=\Omega_{i m, 1}+\Omega_{i m, 2}+2 \Omega_{i m, 3} .
$$

This completes the proof of (i).

The proof of (ii) is exactly the same as that of (iii) of Theorem 2, and hence is omitted here.

Finally we prove (iii). For every $t=1, \ldots, 7$, it follows from a standard result for instrumental variable estimators that

$$
\sqrt{n_{t}}\left(\hat{\beta}_{t}-\beta_{t}\right) \stackrel{d .}{\longrightarrow} N\left(0, \operatorname{Var}\left(U_{i t}\right) A^{-1}\right),
$$

which implies that

$$
\sqrt{n}\left(\hat{\beta}_{t}-\beta_{t}\right) \stackrel{d .}{\longrightarrow} N\left(0, r_{t} \operatorname{Var}\left(U_{i t}\right) A^{-1}\right) .
$$

Moreover, note that $\hat{\beta}_{t}$ only uses the data on those individuals whose diary day is $t$. Since the individuals are drawn independently, $\hat{\beta}_{t}$ is independent of $\hat{\beta}_{\tau}$ for any $t \neq \tau$. This implies that the asymptotic variance of the within estimator $\hat{\beta}_{w n}$ is

$$
\Omega_{w n}=A^{-1} \sum_{t=1}^{7} r_{t} \mathrm{E}\left(U_{i t}^{2}\right)
$$

Lemma 1. Under Assumption 1 5, the daily instrumental variable estimators are consistent. That is, $\hat{\beta}_{t} \stackrel{p .}{\longrightarrow} \beta_{t}$ for $t=1, \ldots, 7$.

Proof. It is a standard result for instrumental variable estimators, so the proof is omitted.

Proof of Theorem 4 . Lemma 1 gives $\hat{\beta}_{t} \stackrel{p .}{\longrightarrow} \beta_{t}$, and the rest of the result follows simply by law of large numbers and the continuous mapping theorem. 


\section{References}

Abrevaya, J. and J. A. Hausman (1999). Semiparametric estimation with mismeasured dependent variables: an application to duration models for unemployment spells. Annales d'Economie et de Statistique, 243-275.

Ahmed, N., M. Brzozowski, and T. F. Crossley (2006). Measurement errors in recall food consumption data. Technical Report WP06/21, IFS Working Papers.

Angrist, J. D. (1991). Grouped-data estimation and testing in simple labor-supply models. Journal of Econometrics 47(2-3), 243-266.

Barrett, G. F. and D. S. Hamermesh (2017). Labor supply elasticities: Overcoming nonclassical measurement error using more accurate hours data. Journal of Human Resources, 1216-8407R1.

Blau, F. D. and L. M. Kahn (2007). Changes in the labor supply behavior of married women: 1980-2000. Journal of Labor Economics 25(3), 393-438.

Blundell, R., A. Duncan, and C. Meghir (1998). Estimating labor supply responses using tax reforms. Econometrica, 827-861.

Blundell, R. and T. MaCurdy (1999). Labor supply: A review of alternative approaches. In Handbook of labor economics, Volume 3, pp. 1559-1695. Elsevier.

Bound, J., C. Brown, and N. Mathiowetz (2001). Measurement error in survey data. In Handbook of econometrics, Volume 5, pp. 3705-3843. Elsevier.

Bound, J., C. C. Brown, G. Duncan, and W. L. Rodgers (1989). Measurement error in cross-sectional and longitudinal labor market surveys: Results from two validation studies. Working Paper 2884, NBER.

Chen, X., H. Hong, and E. Tamer (2005). Measurement error models with auxiliary data. The Review of Economic Studies 72(2), 343-366.

Duncan, G. J. and D. H. Hill (1985). An investigation of the extent and consequences of measurement error in labor-economic survey data. Journal of Labor Economics 3(4), $508-532$.

Fisher, K., J. Gershuny, S. M. Flood, J. G. Roman, and S. L. Hofferth (2018). Multinational time use study extract system: Version 1.2. [dataset], Minneapolis, MN: IPUMS, https://doi.org/10.18128/D062.V1.2. 
Heckman, J. J. (1993). What has been learned about labor supply in the past twenty years? The American Economic Review 83(2), 116-121.

Hofferth, S. L., S. M. Flood, and M. Sobek. (2018). American time use survey data extract builder: Version 2.7. [dataset], College Park, MD: University of Maryland and Minneapolis, MN: IPUMS, https://doi.org/10.18128/D060.V2.7.

Hu, Y. and Y. Sasaki (2015). Closed-form estimation of nonparametric models with nonclassical measurement errors. Journal of Econometrics 185(2), 392-408.

Hu, Y. and S. M. Schennach (2008). Instrumental variable treatment of nonclassical measurement error models. Econometrica 76(1), 195-216.

Juhn, C. and K. M. Murphy (1997). Wage inequality and family labor supply. Journal of labor economics 15(1, Part 1), 72-97.

Juster, F. T. and F. P. Stafford (1991). The allocation of time: Empirical findings, behavioral models, and problems of measurement. Journal of Economic literature 29(2), 471-522.

Kiviet, J. F. and J. Niemczyk (2009). On the limiting and empirical distribution of iv estimators when some of the instruments are invalid. UvA Econometrics Discussion Paper 2006/02.

McClelland, R. and S. Mok (2012). A review of recent research on labor supply elasticities. Working Paper 2012-12, Congressional Budget Office.

Sousa, J. (2014). Estimation of price elasticities of demand for alcohol in the united kingdom. Technical Report 16, HMRC Working Paper. 